\title{
Chemoselective and Sequential Palladium-Catalyzed Couplings for the Generation of Stilbene Libraries via Immobilized Substrates.
}

Carla I. Traficante, ${ }^{\dagger}$ Catherine Fagundez, ${ }^{\ddagger}$ Gloria L. Serra, ${ }^{\ddagger}$ Ernesto G. Mata, ${ }^{\dagger}$ Carina M. L. Delpiccolo ${ }^{\dagger *}$

${ }^{\dagger}$ Instituto de Química Rosario (CONICET-UNR), Facultad de Ciencias Bioquímicas y Farmacéuticas, Universidad Nacional de Rosario, Suipacha 531, 2000 Rosario, Argentina.

¿Laboratorio de Quimica Farmacéutica, (DQO), Facultad de Qúímica, Universidad de la República, Gral. Flores 2124, Montevideo, 11800, Uruguay.

\section{Supporting Information}

Table of Contents

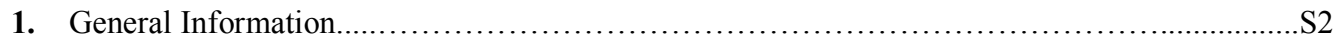

2. General Procedure for Immobilization of Aryl Iodides.........................................................S2

3. General Procedure for the Solid-Phase Hiyama Cross-Coupling Reaction ......................S2

4. General Procedure for the Solid-Phase Heck Cross-Coupling Reaction ....................S2-3

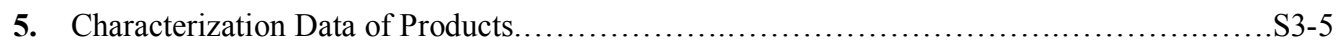

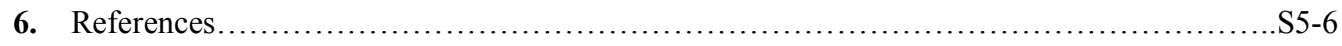

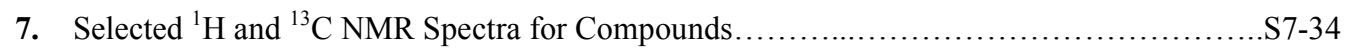




\section{General Information}

Chemical reagents were purchased from commercial sources and were used without further purification unless noted otherwise. Solvents were analytical grade or were purified by standard procedures prior to use. Resins were purchased from Sigma-Aldrich. ${ }^{1} \mathrm{H}$ NMR spectra were recorded in a Bruker Avance spectrometer at $300 \mathrm{MHz}$ in $\mathrm{CDCl}_{3}$ or $\mathrm{CD}_{3} \mathrm{COCD}_{3}$ with tetramethylsilane (TMS) as internal standards.

${ }^{13} \mathrm{C}$ NMR spectra were recorded on the same apparatus at $75 \mathrm{MHz}$ with $\mathrm{CDCl}_{3}$ or $\mathrm{CD}_{3} \mathrm{COCD}_{3}$ as solvents and references. The chemical shifts $(\delta)$ are reported in ppm downfield from TMS and coupling constants $(J)$ are expressed in hertz. Mass spectra were recorded on a Shimadzu QP2010 Plus apparatus at an ionization voltage of $70 \mathrm{eV}$ equipped with a SPBTM-1 capillary column (internal diameter $0.25 \mathrm{~mm}$, length $30 \mathrm{~m}$ ). The high resolution mass spectra were obtained with a Bruker MicroTOF-Q II instrument (Bruker Daltonics, Billerica, MA). Detection of the ions was performed in electrospray ionization, positive ion mode. Solvents were dried using an MBraun solvent system (SPS-800). Analytical thin-layer chromatography (TLC) was carried out with silica gel 60 F254 pre-coated aluminum sheets. Flash column chromatography was performed using Merck silica gel 60 (230-400 mesh). Elution was carried out with hexane-EtOAc mixtures, under positive pressure and employing gradient of solvent polarity techniques.

Solid-phase reactions were carried out in polypropylene cartridges equipped with a frit (Supelco, Bellefonte, PA), unless reflux conditions were required, in those cases standard glassware was used. All solid-phase reaction mixtures were stirred at the slowest rate.

\section{General Procedure for Immobilization of Aryl Iodides}

$0.2 \mathrm{~g}$ of Wang resin $(1.1 \mathrm{mmol} / \mathrm{g}, 0.22 \mathrm{mmol})$ was swelled by gentle stirring in anhydrous DMF $(5 \mathrm{~mL})$. Then, 4-iodobenzoic acid (0.098 g, $0.66 \mathrm{mmol})$, DCC ( $N, N^{\prime}$-diisopropylcarbodiimide) (0.102 mL, 0.66 mmol) and DMAP (catalytic amount) were added. The mixture was stirred overnight at room temperature. After filtration, the resin was sequentially washed with $\mathrm{CH}_{2} \mathrm{Cl}_{2}(3 \times 5 \mathrm{~mL}), \mathrm{DMF}(3 \times 5 \mathrm{~mL})$, EtOAc $(3 \times 5 \mathrm{~mL}), \mathrm{MeOH}(3 \times 5 \mathrm{~mL}), \mathrm{CH}_{2} \mathrm{Cl}_{2}$ and finally dried under high vacuum thus obtaining the immobilized aryl iodide $3\{1\}$. Mass recovery was used to determine resin loading after cleavage of an aliquot with $10 \% \mathrm{TFA} / \mathrm{CH}_{2} \mathrm{Cl}_{2}$. In general, coupling was achieved with greater than $95 \%$ efficiency.

Following this procedure, compound $\mathbf{3}\{2\}$ was also synthesized.

\section{General Procedure for the Solid-Phase Hiyama Cross-Coupling Reaction.}

Support-bound aryl halide $3\{1\}(0.88 \mathrm{mmol} / \mathrm{g}, 0.18 \mathrm{mmol})$ was suspended in anhydrous THF $(5 \mathrm{~mL})$, and were added in sequential order under a nitrogen atmosphere $\mathrm{Pd}\left(\mathrm{PPh}_{3}\right)_{4}(0.020 \mathrm{~g}, 10 \mathrm{~mol} \%)$, vinyltriethoxysilane $1(0.189 \mathrm{~mL}, 0.9 \mathrm{mmol})$ and TBAF (tetra-n-butylammonium fluoride) $(0.9 \mathrm{~mL}, 1 \mathrm{M}$ in THF). The flask was fitted with a condenser and the reaction mixture was stirred $5 \mathrm{~h}$ at $80{ }^{\circ} \mathrm{C}$. Subsequently, the resin was filtered and washed successively with $\mathrm{CH}_{2} \mathrm{Cl}_{2}(3 \times 5 \mathrm{~mL})$, THF $(3 \times 5 \mathrm{~mL})$, $\operatorname{DMF}(3 \times 5 \mathrm{~mL}), \mathrm{MeOH}(3 \times 5 \mathrm{~mL})$ and finally with $\mathrm{CH}_{2} \mathrm{Cl}_{2}$ thus obtaining the support-bound vinyl arene $4\{1\}$. Then, the resin is dry under high vacuum, and use for the Heck Cross-Coupling Reaction.

Following this procedure, compound $\mathbf{4}\{2\}$ was also synthesized.

\section{General Procedure for the Solid-Phase Heck Cross-Coupling Reaction.}

Method A: Support-bound vinyl arene $4\{1\}(0.96 \mathrm{mmol} / \mathrm{g}, 0.19 \mathrm{mmol})$ was suspended in anhydrous DMF $(5 \mathrm{~mL})$, and were added in sequential order under a nitrogen atmosphere, $\operatorname{Pd}_{2}(\mathrm{dba})_{3}(0.018 \mathrm{~g}, 10$ mol\%), P(o-tolyl) $(0.005 \mathrm{~g}, 10 \mathrm{~mol} \%)$, aryl halide $\mathbf{6}\{1\}(0.064 \mathrm{~mL}, 0.57 \mathrm{mmol})$, and TEA (triethylamine) $(133 \mathrm{~mL}, 0.95 \mathrm{mmol})$. The flask was fitted with a condenser and the reaction mixture was stirred $24 \mathrm{~h}$ at $110{ }^{\circ} \mathrm{C}$. Subsequently, the resin was filtered and washed successively with $\mathrm{CH}_{2} \mathrm{Cl}_{2}(3 \times 5 \mathrm{~mL})$, THF $(3 \times 5$ $\mathrm{mL}), \mathrm{DMF}(3 \times 5 \mathrm{~mL}), \mathrm{MeOH}(3 \times 5 \mathrm{~mL})$ and finally with $\mathrm{CH}_{2} \mathrm{Cl}_{2}$. After drying under high vacuum, the compound was cleaved from the support with $5 \mathrm{~mL}$ of a $10 \%$ TFA in $\mathrm{CH}_{2} \mathrm{Cl}_{2}$ for 50 min at room temperature. Then the mixture was filtered, washed with $\mathrm{CH}_{2} \mathrm{Cl}_{2}(2 \times 5 \mathrm{~mL})$ and the filtrate was evaporated under reduced pressure. Esterification with diazomethane afforded the crude product that was analyzed by ${ }^{1} \mathrm{H}$ NMR and GC/MS and then purified by column chromatography (hexane- EtOAc), yielding $20 \mathrm{mg}$ of pure $(E)$-Methyl 4-styrylbenzoate $(\mathbf{8}\{1,1\})(51 \%)$.

Following this methodology, seven more compounds were synthesized: 
$\mathbf{8}\{1,3\}$ (10\% yield), $\mathbf{8}\{1,5\}$ (38\% yield), $8\{1,6\}$ (44\% yield), $8\{1,7\}$ (24\% yield), $8\{1,8\}$ ( $8 \%$ yield), $\mathbf{8}\{1,9\}(21 \%$ yield $), \mathbf{8}\{1,2\}(63 \%$ yield $){ }^{*}$

Method B: Support-bound vinyl arene $4\{1\}(0.96 \mathrm{mmol} / \mathrm{g}, 0.19 \mathrm{mmol})$ was suspended in anhydrous DMF $(5 \mathrm{~mL})$, and were added in sequential order under a nitrogen atmosphere, $\mathrm{Pd}(\mathrm{OAc})_{2}(0.021 \mathrm{~g}, 50$ mol \%), $\mathrm{Bu}_{4} \mathrm{NCl}$ (tetra-n-butylammonium chloride) $(0.026 \mathrm{~g}, 0.095 \mathrm{mmol})$, aryl halide $6\{3\}(0.207 \mathrm{~g}$, $0.95 \mathrm{mmol})$ and TEA $(0.267 \mathrm{~mL}, 1.9 \mathrm{mmol})$. The flask was fitted with a condenser and the reaction mixture was stirred $18 \mathrm{~h}$ at $110^{\circ} \mathrm{C}$. Subsequently, the resin was filtered and washed successively with $\mathrm{CH}_{2} \mathrm{Cl}_{2}(3 \times 5 \mathrm{~mL})$, THF ( $\left.3 \times 5 \mathrm{~mL}\right)$, DMF $(3 \times 5 \mathrm{~mL}), \mathrm{MeOH}(3 \times 5 \mathrm{~mL})$ and finally with $\mathrm{CH}_{2} \mathrm{Cl}_{2}$. After drying under high vacuum, the compound was cleaved from the support with $5 \mathrm{~mL}$ of a $10 \%$ TFA in $\mathrm{CH}_{2} \mathrm{Cl}_{2}$ for $50 \mathrm{~min}$ at room temperature. Then the mixture was filtered, washed with $\mathrm{CH}_{2} \mathrm{Cl}_{2}(2 \times 5 \mathrm{~mL})$ and the filtrate was evaporated under reduced pressure. Esterification with diazomethane afforded the crude product that was analyzed by ${ }^{1} \mathrm{H}$ NMR and GC/MS and then purified by column chromatography (hexane- EtOAc) yielding $17 \mathrm{mg}$ of pure $(E)$-Methyl 4-(4-methylstyryl)benzoate $(\mathbf{8}\{1,3\})(40 \%)$.

Following this methodology, ten more compounds were synthesized:

$\mathbf{8}\{2,1\}$ (36\% yield), $\mathbf{8}\{1,4\}$ (65\% yield), $8\{2,4\}$ (30\% yield), $8\{1,2\}$ (47\% yield), $8\{2,2\}$ (79\% yield), $\mathbf{8}\{2,3\}$ (21\% yield), $\mathbf{8}\{1,8\}$ (46\% yield), $8\{1,9\}$ (15\% yield), $8\{1,10\}$ (19\% yield), $8\{1,11\}(10 \%$ yield).

\section{Characterization data of products}

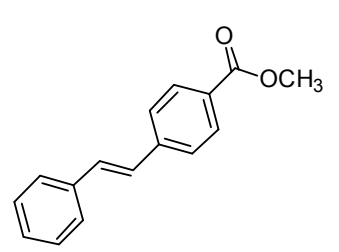

(E)-Methyl 4-styrylbenzoate $(8\{1,1\}):^{1}$

${ }^{1} \mathrm{H} \mathrm{NMR}\left(\mathrm{CDCl}_{3}, 300 \mathrm{MHz}\right) \delta: 3.93$ (s, 3H), 7.12 (d, $\left.J=16.4 \mathrm{~Hz}, 1 \mathrm{H}\right), 7.17$ (d, $J=16.4 \mathrm{~Hz}, 1 \mathrm{H}), 7.31(\mathrm{~d}, J=8.4 \mathrm{~Hz}, 1 \mathrm{H}), 7.38(\mathrm{t}, J=8.4 \mathrm{~Hz}, 2 \mathrm{H}), 7.55(\mathrm{t}, J=$ $8.4 \mathrm{~Hz}, 4 \mathrm{H}), 8.02(\mathrm{~d}, J=8.4 \mathrm{~Hz}, 2 \mathrm{H}) .{ }^{13} \mathrm{C} \mathrm{NMR}\left(\mathrm{CDCl}_{3}, 75 \mathrm{MHz}\right) \delta: 52.1$, $126.3,126.8,127.6,128.2,128.8,128.9,130.0,131.2,136.7,141.8,166.9$.

GC/MS: $\mathrm{t}_{\mathrm{R}} 22.26 \mathrm{~min}\left(50^{\circ} \mathrm{C}(3 \mathrm{~min}), 10^{\circ} \mathrm{C} / \mathrm{min}, 300^{\circ} \mathrm{C}, 49.6 \mathrm{kPa}\right), \mathrm{m} / \mathrm{z}(\%) 238\left(\mathrm{M}^{+}, 100\right)$.

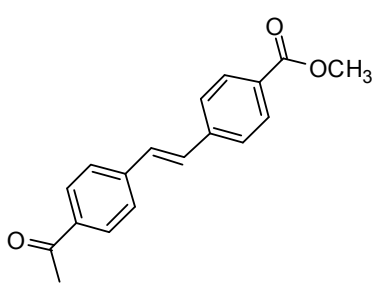

(E)-Methyl 4-(4-acetylstyryl)benzoate $(8\{1,2\})$

${ }^{1} \mathrm{H}$ NMR $\left(\mathrm{CDCl}_{3}, 300 \mathrm{MHz}\right) \delta: 2.62$ (s, 3H), 3.93 (s, 3H), 7.24 (s, 2H), $7.59(\mathrm{~d}, J=8.3 \mathrm{~Hz}, 2 \mathrm{H}), 7.61(\mathrm{~d}, J=8.3 \mathrm{~Hz}, 2 \mathrm{H}), 7.97$ (d, $J=8.6 \mathrm{~Hz}, 2 \mathrm{H})$, $8.04(\mathrm{~d}, J=8.6 \mathrm{~Hz}, 2 \mathrm{H}) .{ }^{13} \mathrm{C} \mathrm{NMR}\left(\mathrm{CDCl}_{3}, 75 \mathrm{MHz}\right) \delta: 26.6,52.1,126.6$, 126.8, 128.9, 129.5, 129.9, 130.1, 130.2, 136.4, 141.1, 141.3, 166.7, 197.4. GC/MS: $\mathrm{t}_{\mathrm{R}} 25.73 \mathrm{~min}\left(50^{\circ} \mathrm{C}(3 \mathrm{~min}), 10{ }^{\circ} \mathrm{C} / \mathrm{min}, 300^{\circ} \mathrm{C}, 49.6 \mathrm{kPa}\right), \mathrm{m} / \mathrm{z}$ (\%) $280\left(\mathrm{M}^{+}\right), 265(100)$.

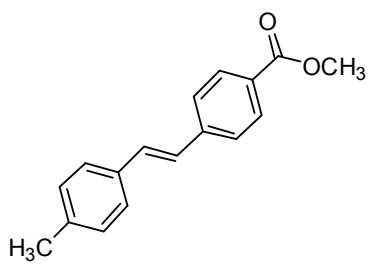

(E)-Methyl 4-(4-methylstyryl)benzoate $(8\{1,3\}):^{2}$

${ }^{1} \mathrm{H}$ NMR $\left(\mathrm{CDCl}_{3}, 300 \mathrm{MHz}\right) \delta: 2.37$ (s, 3H), 3.92 (s, 3H), 7.05-7.23 (m, 4H), $7.43(\mathrm{~d}, J=8.1 \mathrm{~Hz}, 2 \mathrm{H}), 7.55(\mathrm{~d}, J=8.3 \mathrm{~Hz}, 2 \mathrm{H}), 8.02(\mathrm{~d}, J=8.3 \mathrm{~Hz}$, $2 \mathrm{H}) .{ }^{13} \mathrm{C} \mathrm{NMR}\left(\mathrm{CDCl}_{3}, 75 \mathrm{MHz}\right) \delta: 21.3,52.1,126.2,126.6,126.7,128.7$, $129.5,130.0,131.2,133.9,138.3,142.1,166.9$. GC/MS: $t_{R} 23.4 \min \left(50^{\circ} \mathrm{C}\right.$ (3 min), $\left.10{ }^{\circ} \mathrm{C} / \mathrm{min}, 300^{\circ} \mathrm{C}, 49.6 \mathrm{kPa}\right), \mathrm{m} / \mathrm{z}(\%) 252\left(\mathrm{M}^{+}, 100\right)$.

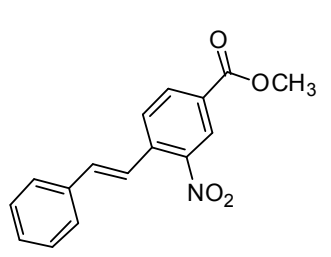

(E)-Methyl 3-nitro-4-styrylbenzoate $(8\{2,1\})$ :

${ }^{1} \mathrm{H}$ NMR $\left(\mathrm{CDCl}_{3}, 300 \mathrm{MHz}\right) \delta: 3.98$ (s, 3H), 7.21 (d, $\left.J=16.7 \mathrm{~Hz}, 1 \mathrm{H}\right), 7.35-$ 7.44 (m, 3H), 7.55-7.64 (m, 3H), 7.86 (d, $J=8.1 \mathrm{~Hz}, 1 \mathrm{H}), 8.23$ (dd, $J=8.1,1.7$ $\mathrm{Hz}, 1 \mathrm{H}), 8.59$ (d, $J=1.7 \mathrm{~Hz}, 1 \mathrm{H}) .{ }^{13} \mathrm{C} \mathrm{NMR}\left(\mathrm{CDCl}_{3}, 75 \mathrm{MHz}\right) \delta: 52.7,122.4$, $126.1,127.4,128.1,128.9,129.2,129.8,133.4,136.0,136.9,147.9,164.9$.

\footnotetext{
*Yield calculated from the crude mixture.
} 
HRMS (ESI) m/z $306.07353[(\mathrm{M}+\mathrm{Na})]^{+}$; calcd for: $\mathrm{C}_{16} \mathrm{H}_{13} \mathrm{NNaO}_{4} 306.07368$.

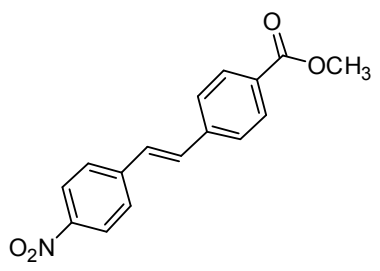

(E)-Methyl 4-(4-nitrostyryl)benzoate $(8\{1,4\}):^{3}$

${ }^{1} \mathrm{H}$ NMR $\left(\mathrm{CDCl}_{3}, 300 \mathrm{MHz}\right) \delta: 3.94$ (s, 3H), 7.27 (s, 2H), 7.60 (d, $J=8.5$

$\mathrm{Hz}, 2 \mathrm{H}), 7.66$ (d, $J=8.5 \mathrm{~Hz}, 2 \mathrm{H}), 8.06$ (d, $J=8.5 \mathrm{~Hz}, 2 \mathrm{H}), 8.24$ (d, $J=8.5$

$\mathrm{Hz}, 2 \mathrm{H}) .{ }^{13} \mathrm{C} \mathrm{NMR}\left(\mathrm{CDCl}_{3}, 75 \mathrm{MHz}\right) \delta: 52.2,124.2,126.9,127.2,128.7$,

$130.0,130.2,132.1,140.5,143.2,147.2,166.6 . \mathrm{GC} / \mathrm{MS}: \mathrm{t}_{\mathrm{R}} 26.48 \mathrm{~min}$ $\left(50^{\circ} \mathrm{C}(3 \mathrm{~min}), 10^{\circ} \mathrm{C} / \mathrm{min}, 300^{\circ} \mathrm{C}, 49.6 \mathrm{kPa}\right), \mathrm{m} / \mathrm{z}(\%) 283\left(\mathrm{M}^{+}, 100\right)$.

(E)-Methyl 4-(4-formylstyryl)benzoate $(8\{1,5\})$ :

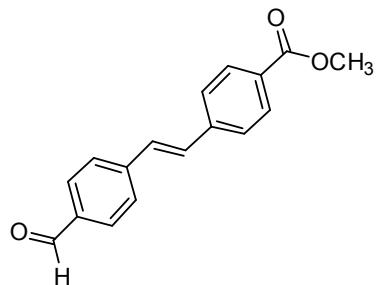

${ }^{1} \mathrm{H}$ NMR $\left(\mathrm{CDCl}_{3}, 300 \mathrm{MHz}\right) \delta: 3.93$ (s, 3H), 7.27 (s, 2H), 7.60 (d, $J=8.4$

$\mathrm{Hz}, 2 \mathrm{H}), 7.68$ (d, $J=8.4 \mathrm{~Hz}, 2 \mathrm{H}), 7.89$ (d, $J=8.4 \mathrm{~Hz}, 2 \mathrm{H}), 8.05$ (d, $J=8.4$

$\mathrm{Hz}, 2 \mathrm{H}), 10.01$ (s, 1H). ${ }^{13} \mathrm{C} \mathrm{NMR}\left(\mathrm{CDCl}_{3}, 75 \mathrm{MHz}\right) \delta: 52.2,126.7,127.2$, $129.7,129.8,130.1,130.3,130.9,135.7,140.9,142.7,166.7,191.5$. HRMS (ESI) $\mathrm{m} / \mathrm{z} 289.08316 \quad[(\mathrm{M}+\mathrm{Na})]^{+}$; calcd for $\mathrm{C}_{17} \mathrm{H}_{14} \mathrm{NaO}_{3}$ :

289.08352 .

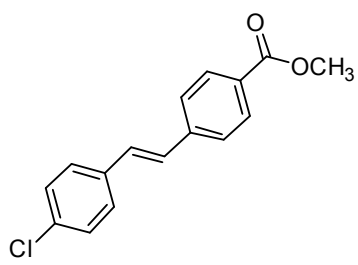

(E)-Methyl 4-(4-chlorostyryl)benzoate $(8\{1,6\}):^{4}$

${ }^{1} \mathrm{H}$ NMR $\left(\mathrm{CDCl}_{3}, 300 \mathrm{MHz}\right) \delta: 3.92(\mathrm{~s}, 3 \mathrm{H}), 7.08(\mathrm{~d}, J=16.4 \mathrm{~Hz}, 1 \mathrm{H}), 7.16$ $(\mathrm{d}, J=16.4 \mathrm{~Hz}, 1 \mathrm{H}), 7.34(\mathrm{~d}, J=8.4 \mathrm{~Hz}, 2 \mathrm{H}), 7.46(\mathrm{~d}, J=8.4 \mathrm{~Hz}, 2 \mathrm{H}), 7.56$ $(\mathrm{d}, J=8.4 \mathrm{~Hz}, 2 \mathrm{H}), 8.03(\mathrm{~d}, J=8.4 \mathrm{~Hz}, 2 \mathrm{H}) .{ }^{13} \mathrm{C} \mathrm{NMR}\left(\mathrm{CDCl}_{3}, 75 \mathrm{MHz}\right) \delta$ : $52.1,126.4,127.9,128.2,128.9,129.1,129.9,130.1,133.9,135.3,141.4$, 166.8. GC/MS: $\mathrm{t}_{\mathrm{R}} 23.7 \mathrm{~min}\left(50^{\circ} \mathrm{C}(3 \mathrm{~min}), 10^{\circ} \mathrm{C} / \mathrm{min}, 300^{\circ} \mathrm{C}, 49.6 \mathrm{kPa}\right), \mathrm{m} / \mathrm{z}(\%) 272\left(\mathrm{M}^{+}, 100\right)$.

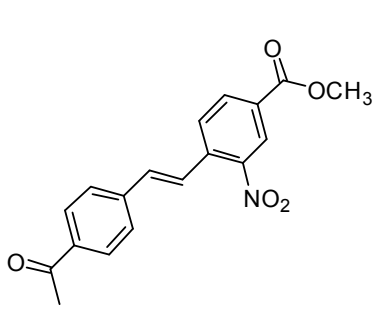

(E)-Methyl 4-(4-acetylstyryl)-3-nitrobenzoate $(8\{2,2\})$ :

${ }^{1} \mathrm{H} \mathrm{NMR}\left(\mathrm{CDCl}_{3}, 300 \mathrm{MHz}\right) \delta: 2.62(\mathrm{~s}, 3 \mathrm{H}), 3.98$ (s, 3H), 7.21 (d, $J=16.2$ $\mathrm{Hz}, 1 \mathrm{H}), 7.62$ (d, $J=8.3 \mathrm{~Hz}, 2 \mathrm{H}), 7.70(\mathrm{~d}, J=16.2 \mathrm{~Hz}, 1 \mathrm{H}), 7.86$ (d, $J=$ $8.3 \mathrm{~Hz}, 1 \mathrm{H}), 7.97(\mathrm{~d}, J=8.3 \mathrm{~Hz}, 2 \mathrm{H}), 8.24(\mathrm{dd}, J=8.3,1.7 \mathrm{~Hz}, 1 \mathrm{H}), 8.61$ $(\mathrm{d}, J=1.7 \mathrm{~Hz}, 1 \mathrm{H}) .{ }^{13} \mathrm{C} \mathrm{NMR}\left(\mathrm{CDCl}_{3}, 75 \mathrm{MHz}\right) \delta: 26.7,52.8,125.2$, $126.2,126.8,127.4,128.4,128.9,130.4,133.6,134.5,136.3,137.1,140.4$, 147.9, 164.8, 197.4. HRMS (ESI) m/z 348.08331 [(M+ Na)] ${ }^{+}$; calcd for $\mathrm{C}_{18} \mathrm{H}_{15} \mathrm{NNaO}_{5}$ : 348.08424 .

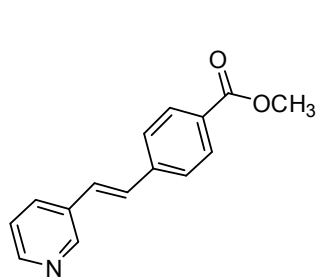

(E)-Methyl 4-(2-(pyridin-3-yl)vinyl)benzoate $(8\{1,7\}):^{5}$

${ }^{1} \mathrm{H} \mathrm{NMR}\left(\mathrm{CDCl}_{3}, 300 \mathrm{MHz}\right) \delta: 3.94$ (s, 3H), 7.19 (s, 2H), 7.32 (dd, $J=7,8 \mathrm{~Hz}$, $J=4,6 \mathrm{~Hz}, 1 \mathrm{H}), 7.59(\mathrm{~d}, J=8.4 \mathrm{~Hz}, 2 \mathrm{H}), 7.86(\mathrm{dt}, J=7,8 \mathrm{~Hz}, J=1.9 \mathrm{~Hz}, 1 \mathrm{H})$, $8.05(\mathrm{~d}, J=8.4 \mathrm{~Hz}, 2 \mathrm{H}), 8.53(\mathrm{da}, J=4,6 \mathrm{~Hz}, 1 \mathrm{H}), 8.75(\mathrm{sa}, 1 \mathrm{H}) .{ }^{13} \mathrm{C} \mathrm{NMR}$ $\left(\mathrm{CDCl}_{3}, 75 \mathrm{MHz}\right) \delta: 52.1,123.7,126.5,127.4,129.5,129.7,130.1,132.9$, 141.0, 148.7, 149.0, 166.7. GC/MS: $\mathrm{t}_{\mathrm{R}} 22.4 \mathrm{~min}\left(50^{\circ} \mathrm{C}(3 \mathrm{~min}), 10^{\circ} \mathrm{C} / \mathrm{min}, 300^{\circ} \mathrm{C}, 49.6 \mathrm{kPa}\right), \mathrm{m} / \mathrm{z}(\%)$ $239\left(\mathrm{M}^{+}\right), 238(100)$.

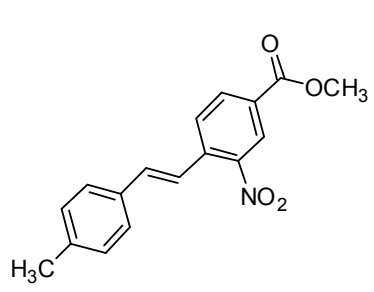

(E)-Methyl 4-(4-methylstyryl)-3-nitrobenzoate (8 $\{2,3\})$ :

${ }^{1} \mathrm{H}$ NMR $\left(\mathrm{CDCl}_{3}, 300 \mathrm{MHz}\right) \delta: 2.38$ (s, 3H), 3.97 (s, 3H), 7.19 (d, $J=15.8$ $\mathrm{Hz}, 1 \mathrm{H}), 7.21(\mathrm{~d}, J=8.2 \mathrm{~Hz}, 2 \mathrm{H}), 7.45(\mathrm{~d}, J=8.2 \mathrm{~Hz}, 2 \mathrm{H}), 7.55(\mathrm{~d}, J=$ $15.8 \mathrm{~Hz}, 1 \mathrm{H}), 7.86(\mathrm{~d}, J=8.3 \mathrm{~Hz}, 1 \mathrm{H}), 8.21(\mathrm{dd}, J=1.7,8.3 \mathrm{~Hz}, 1 \mathrm{H}), 8.58$ $(\mathrm{d}, J=1.7 \mathrm{~Hz}, 1 \mathrm{H}) .{ }^{13} \mathrm{C} \mathrm{NMR}\left(\mathrm{CDCl}_{3}, 75 \mathrm{MHz}\right) \delta: 21.4,52.7,121.3,126.1$, 127.3, 127.9, 129.5, 129.6, 133.3, 136.1, 137.0, 139.5, 147.8, 164.9. HRMS 
(ESI) $\mathrm{m} / \mathrm{z} 320.08965[(\mathrm{M}+\mathrm{Na})]^{+}$; calcd for $\mathrm{C}_{17} \mathrm{H}_{15} \mathrm{NNaO}_{4}: 320.08933$.

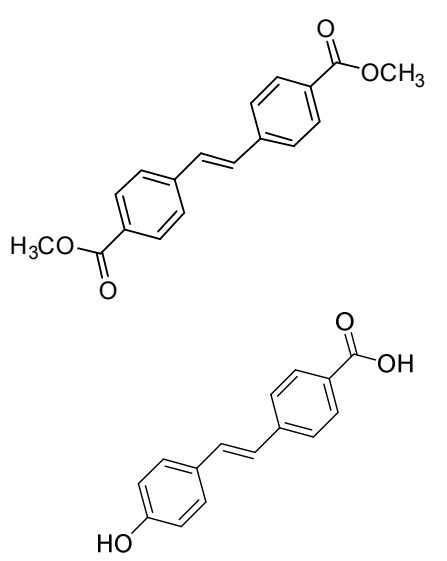

(E)-Dimethyl 4,4'-(ethene-1,2-diyl)dibenzoate $(8\{1,8\}){ }^{6}$

${ }^{1} \mathrm{H} \mathrm{NMR}\left(\mathrm{CDCl}_{3}, 300 \mathrm{MHz}\right) \delta: 3.96$ (s, 3H), 7.23 (s, 2H), $7.58(\mathrm{~d}, J=$ $8.3 \mathrm{~Hz}, 4 \mathrm{H}), 8.04(\mathrm{~d}, J=8.3 \mathrm{~Hz}, 4 \mathrm{H}) .{ }^{13} \mathrm{C} \mathrm{NMR}\left(\mathrm{CDCl}_{3}, 75 \mathrm{MHz}\right) \delta$ : $52.1,126.6,129.5,130.0,130.1,141.2,166.8$. GC/MS: $t_{R} 26.23 \mathrm{~min}$ $\left(50^{\circ} \mathrm{C}(3 \mathrm{~min}), 10^{\circ} \mathrm{C} / \mathrm{min}, 300^{\circ} \mathrm{C}, 49.6 \mathrm{kPa}\right), \mathrm{m} / \mathrm{z}(\%) 296\left(\mathrm{M}^{+}, 100\right)$. (E)-4-(4-Hydroxystyryl)benzoic acid $(9\{1,9\})^{\dagger}$ :

${ }^{1} \mathrm{H} \mathrm{NMR}\left(\mathrm{CD}_{3} \mathrm{COCD}_{3}, 300 \mathrm{MHz}\right) \delta: 6.86(\mathrm{~d}, J=8.7 \mathrm{~Hz}, 2 \mathrm{H}), 7.12(\mathrm{~d}, J$ $=16.4 \mathrm{~Hz}, 1 \mathrm{H}), 7.34(\mathrm{~d}, J=16.4 \mathrm{~Hz}, 1 \mathrm{H}), 7.5(\mathrm{~d}, J=8.7 \mathrm{~Hz}, 2 \mathrm{H}), 7.66$ $(\mathrm{d}, J=8.3 \mathrm{~Hz}, 2 \mathrm{H}), 7.99$ (d, $J=8.3 \mathrm{~Hz}, 2 \mathrm{H}) .{ }^{13} \mathrm{C} \mathrm{NMR}\left(\mathrm{CD}_{3} \mathrm{COCD}_{3}, 75\right.$ MHz) $\delta: 115.5,124.4,125.9,128.3,128.6,129.9,131.2,142.6,157.8$, 166.5. HRMS (ESI) m/z 239.07149 [(M-H)]'; calcd for $\mathrm{C}_{15} \mathrm{H}_{11} \mathrm{O}_{3}: 239.07137$.

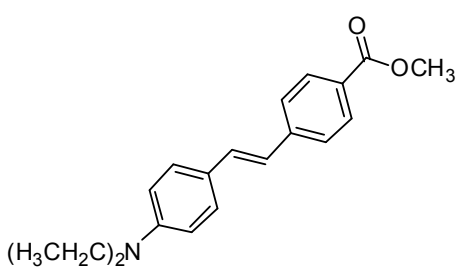

(E)-Methyl 4-(4-(diethylamino)styryl)benzoate $(8\{1,10\})$ :

${ }^{1} \mathrm{H} \mathrm{NMR}\left(\mathrm{CDCl}_{3}, 300 \mathrm{MHz}\right) \delta: 1.19(\mathrm{t}, J=7.0 \mathrm{~Hz}, 6 \mathrm{H}), 3.39$ (q, $J=$ $7.1 \mathrm{~Hz}, 4 \mathrm{H}), 6.66(\mathrm{~d}, J=8.8 \mathrm{~Hz}, 2 \mathrm{H}), 6.89(\mathrm{~d}, J=16.2 \mathrm{~Hz}, 1 \mathrm{H})$, 7.14 (d, $J=16.2 \mathrm{~Hz}, 1 \mathrm{H}), 7.40$ (d, $J=8.8 \mathrm{~Hz}, 2 \mathrm{H}), 7.50$ (d, $J=8.4$ $\mathrm{Hz}, 2 \mathrm{H}), 7.98$ (d, $J=8.4 \mathrm{~Hz}, 2 \mathrm{H}) .{ }^{13} \mathrm{C} \mathrm{NMR}\left(\mathrm{CD}_{3} \mathrm{COCD}_{3}, 75 \mathrm{MHz}\right)$ $\delta: 12.6,44.4,51.9,111.6,122.4,123.9,125.6,127.7,128.3,129.9,131.5,143.0,147.9,167.1$. HRMS (ESI) $\mathrm{m} / \mathrm{z} 310.18011[(\mathrm{M}+\mathrm{H})]^{+}$; calcd for $\mathrm{C}_{20} \mathrm{H}_{24} \mathrm{NO}_{2}: 310.18016$.

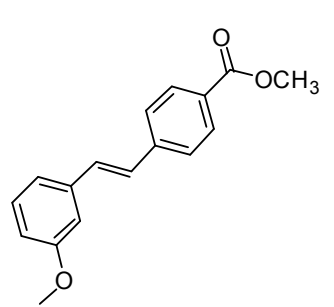

(E)-Methyl 4-(3-methoxystyryl)benzoate $(8\{1,11\}):^{7}$

${ }^{1} \mathrm{H}$ NMR $\left(\mathrm{CDCl}_{3}, 300 \mathrm{MHz}\right) \delta: 3.86(\mathrm{~s}, 3 \mathrm{H}), 3.92(\mathrm{~s}, 3 \mathrm{H}), 6.86(\mathrm{dd}, J=8.0$, $2.38 \mathrm{~Hz}, 1 \mathrm{H}), 7.06-7.22(\mathrm{~m}, 4 \mathrm{H}), 7.30(\mathrm{t}, J=8.0 \mathrm{~Hz}, 1 \mathrm{H}), 7.56(\mathrm{~d}, J=8.4 \mathrm{~Hz}$, $2 \mathrm{H}), 8.02(\mathrm{~d}, J=8.4 \mathrm{~Hz}, 2 \mathrm{H}) .{ }^{13} \mathrm{C} \mathrm{NMR}\left(\mathrm{CDCl}_{3}, 75 \mathrm{MHz}\right) \delta: 52.1,55.3,112.0$, $113.9,119.5,126.4,127.9,128.9,129.3,129.8,131.1,138.20,141.7,159.9$, 166.9. GC/MS: $\mathrm{t}_{\mathrm{R}} 24.05 \mathrm{~min}\left(50^{\circ} \mathrm{C}(3 \mathrm{~min}), 10{ }^{\circ} \mathrm{C} / \mathrm{min}, 300^{\circ} \mathrm{C}, 49.6 \mathrm{kPa}\right), \mathrm{m} / \mathrm{z}$

(\%) $268\left(\mathrm{M}^{+}, 100\right)$.

\section{References}

1. Tian, J.; Moeller, K. D., Electrochemically Assisted Heck Reactions. Org. Lett. 2005, 7, 53815383.

2. Poeylaut-Palena, A. A.; Testero, S. A.; Mata, E. G., Solid-Supported Cross Metathesis and the Role of the Homodimerization of the Non-Immobilized Olefin. J. Org. Chem. 2008, 73, 2024-2027.

3. Durantini, E. N., Synthesis of Functional Olefins Using the Wittig-Horner Reaction in Different Media. Synth. Commun. 1999, 29, 4201-4222.

4. Pavia, C.; Giacalone, F.; Bivona, L. A.; Salvo, A. M. P.; Petrucci, C.; Strappaveccia, G.; Vaccaro, L.; Aprile, C.; Gruttadauria, M., Evidences of Release and Catch Mechanism in the Heck Reaction Catalyzed by Palladium Immobilized on Highly Cross-linked-supported Imidazolium Salts. $J$. Mol. Catal. A: Chem. 2014, 387, 57-62.

\footnotetext{
${ }^{\dagger}$ Compound $\mathbf{8}\{1,9\}$ was identified as its carboxylic acid $\mathbf{9}\{1,9\}$ to avoid methylation of the $-\mathrm{OH}$ group.
} 
5. Aun, C. E.; Clarkson, T. J.; Happer, D. A. R., The Investigation of $\pi$ Polarization Effects in Stilbazoles, Stilbazole Methiodides, and Styrylfurans by ${ }^{13} \mathrm{C}$ NMR Spectroscopy. J. Chem. Soc., Perkin Trans. 2 1990, 12, 635-643.

6. Dieguez, H. R.; Lopez, A.; Domingo, V.; Arteaga, J. F.; Dobado, J. A.; Herrador, M. M.; Quilez del Moral, J. F.; Barrero, A. F., Weakening C-O Bonds: Ti(III), a New Reagent for Alcohol Deoxygenation and Carbonyl Coupling Olefination. J. Am. Chem. Soc. 2010, 132, 254-259.

7. Anthony, N. G.; Breen, D.; Clarke, J.; Donoghue, G.; Drummond, A. J.; Ellis, E. M.; Gemmell, C. G.; Helesbeux, J. J.; Hunter, I. S.; Khalaf, A. I.; Mackay, S. P.; Parkinson, J. A.; Suckling, C. J.; Waigh, R. D., Antimicrobial Lexitropsins Containing Amide, Amidine, and Alkene Linking Groups. $J$. Med. Chem. 2007, 50, 6116-6125. 
${ }^{1} \mathrm{H}$ NMR of compound $8\{1,1\}\left(\mathrm{CDCl}_{3}, 300 \mathrm{MHz}\right)$

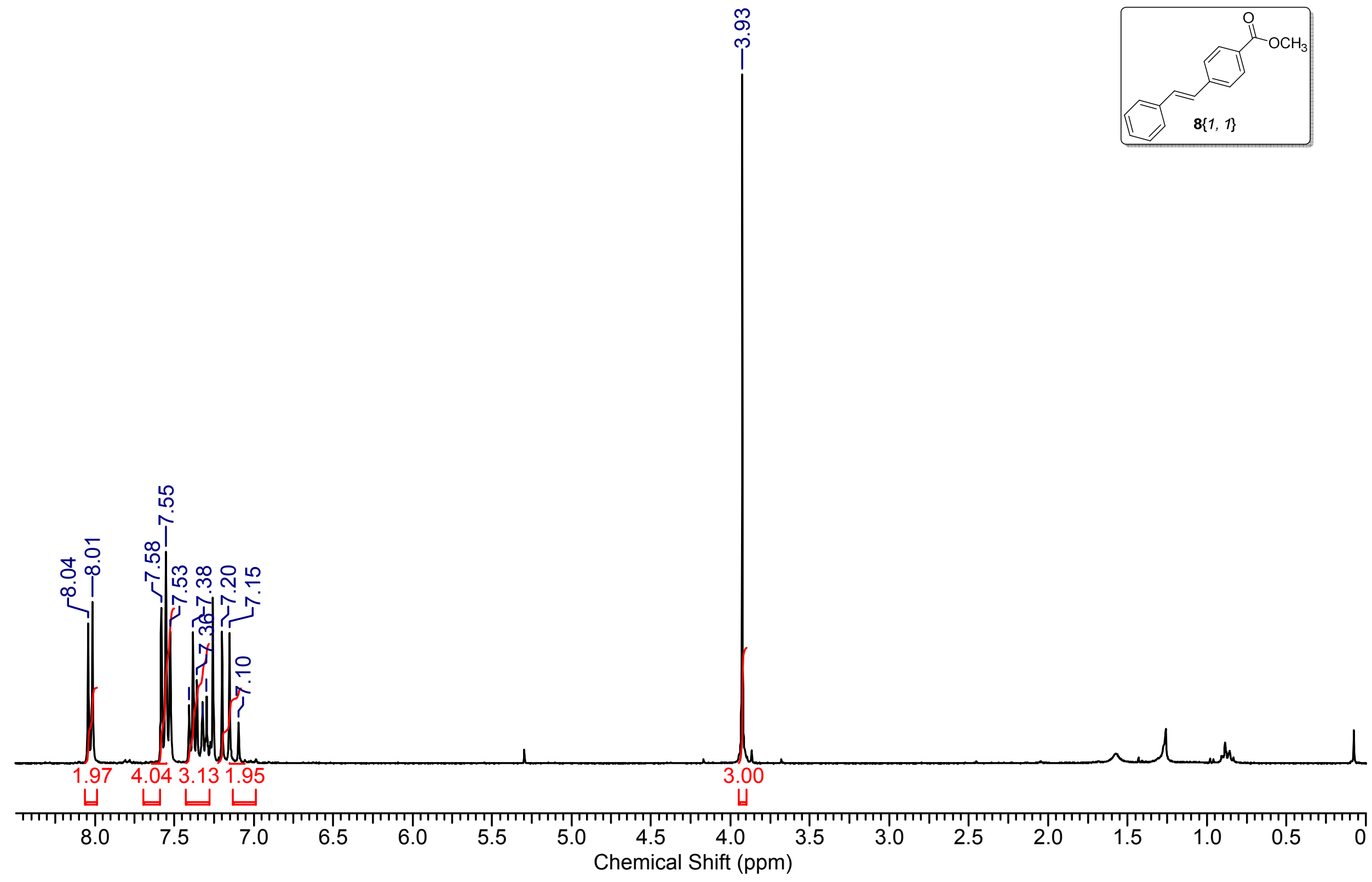


${ }^{13} \mathrm{CNMR}$ of compound $\mathbf{8}\{1,1\}\left(\mathrm{CDCl}_{3}, 75 \mathrm{MHz}\right)$

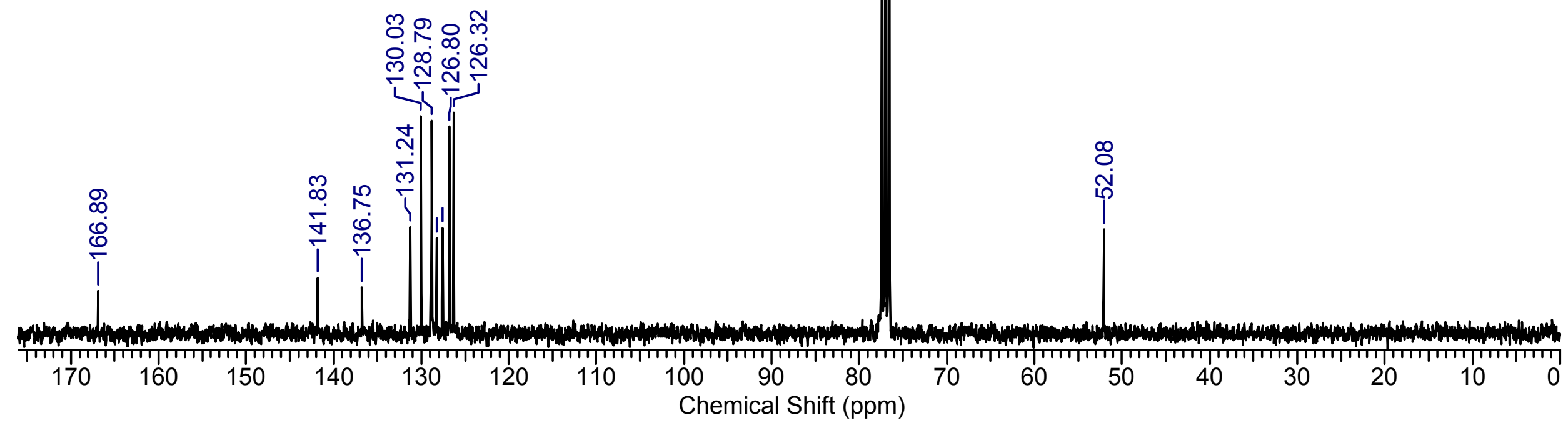


${ }^{1} \mathrm{H}$ NMR of compound $8\{1,2\}\left(\mathrm{CDCl}_{3}, 300 \mathrm{MHz}\right)$

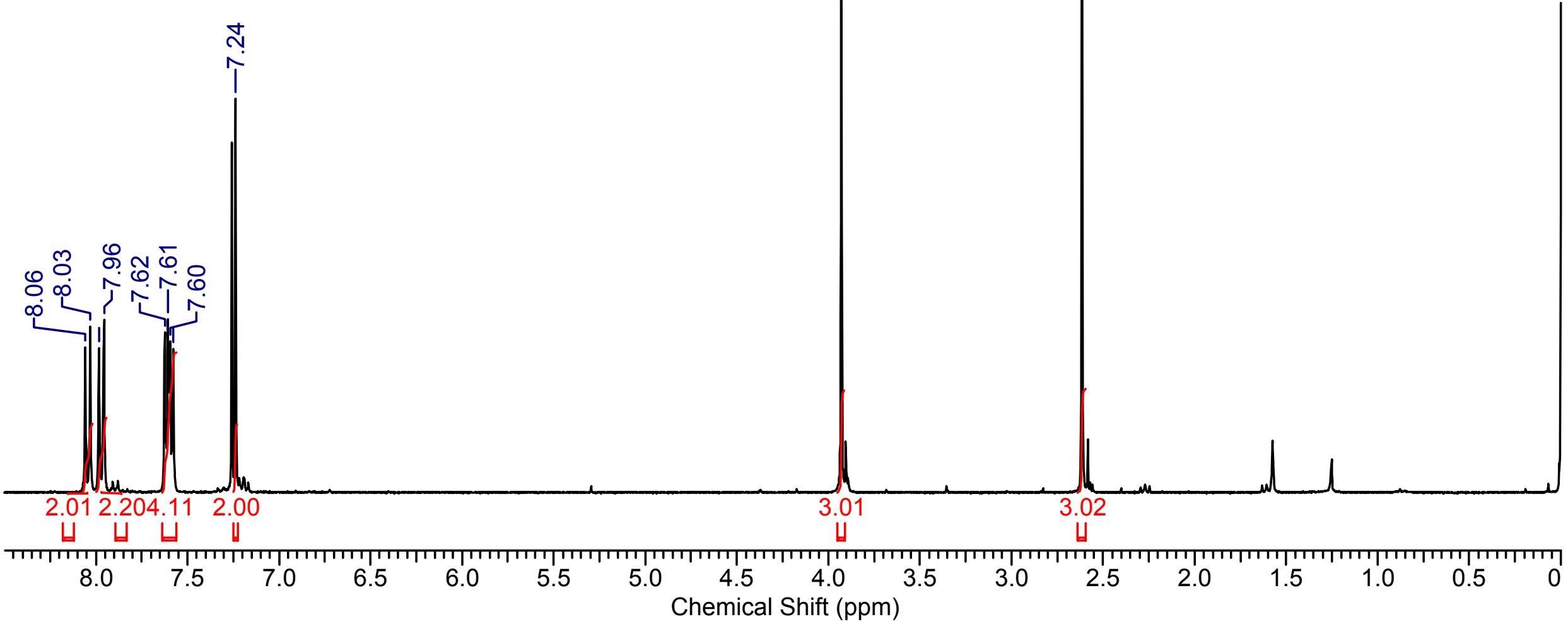


${ }^{13} \mathrm{CNMR}$ of compound $\mathbf{8}\{1,2\}\left(\mathrm{CDCl}_{3}, 75 \mathrm{MHz}\right)$

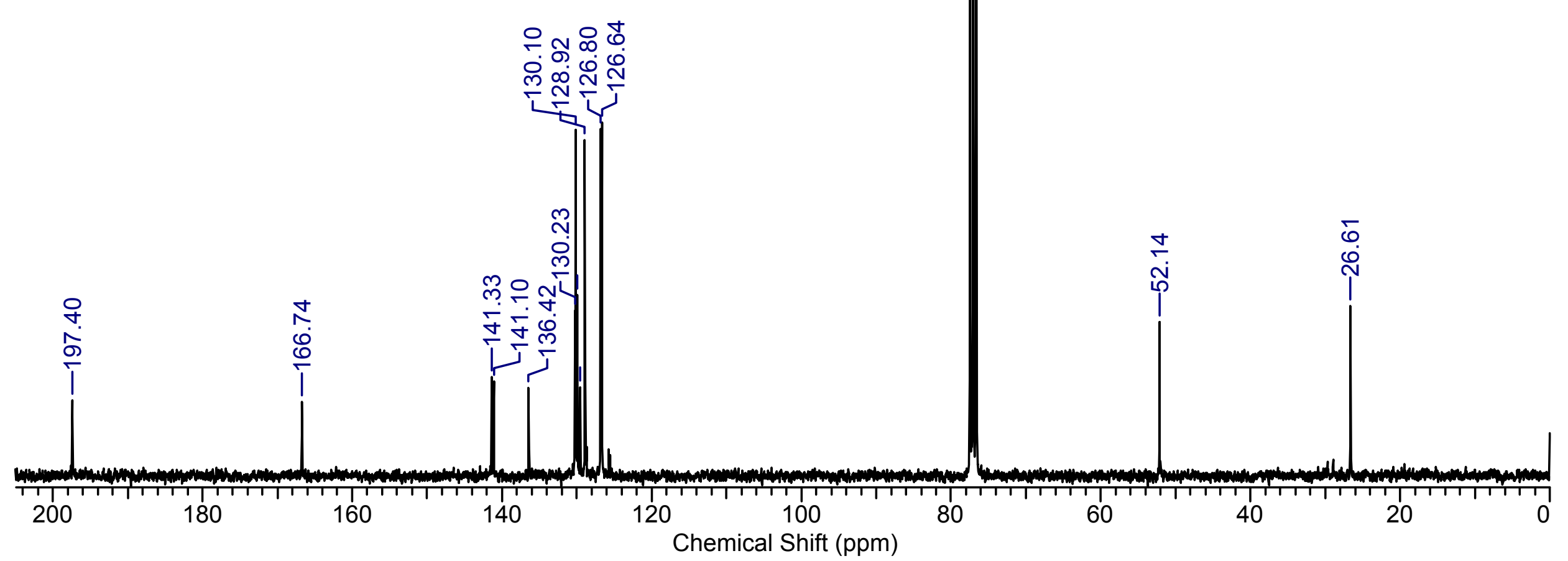


${ }^{1} \mathrm{H}$ NMR of compound $8\{1,3\}\left(\mathrm{CDCl}_{3}, 300 \mathrm{MHz}\right)$

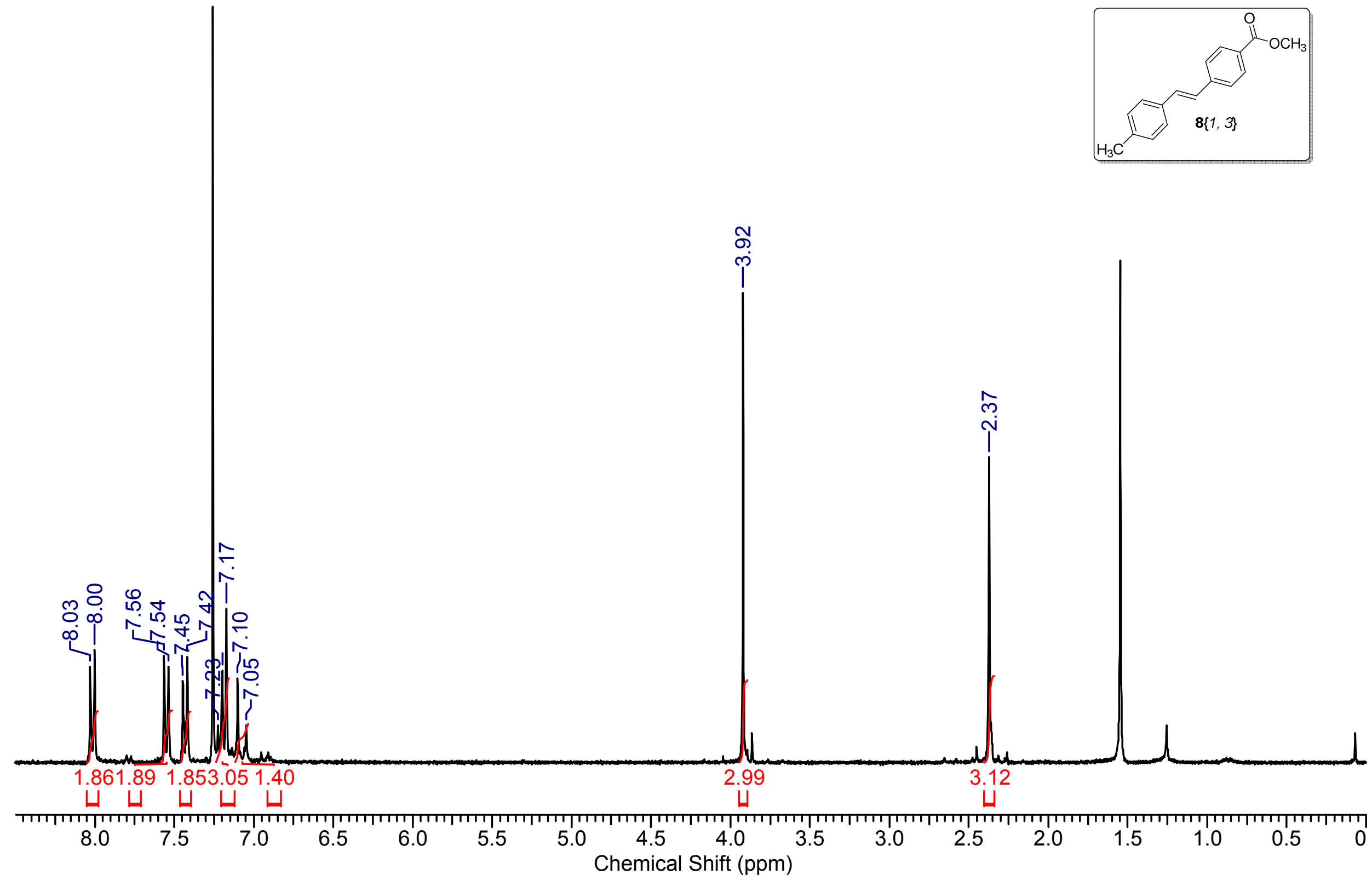


${ }^{13} \mathrm{C}$ NMR of compound $8\{1,3\}\left(\mathrm{CDCl}_{3}, 75 \mathrm{MHz}\right)$

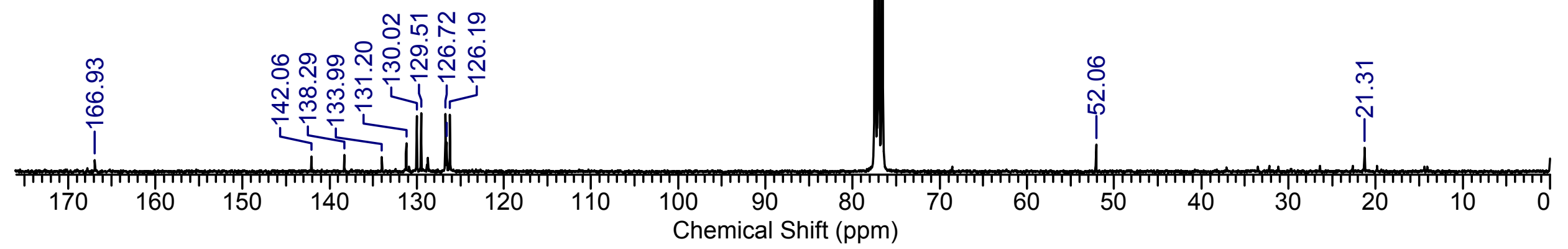


${ }^{1} \mathrm{H}$ NMR of compound $\mathbf{8}\{2,1\}\left(\mathrm{CDCl}_{3}, 300 \mathrm{MHz}\right)$

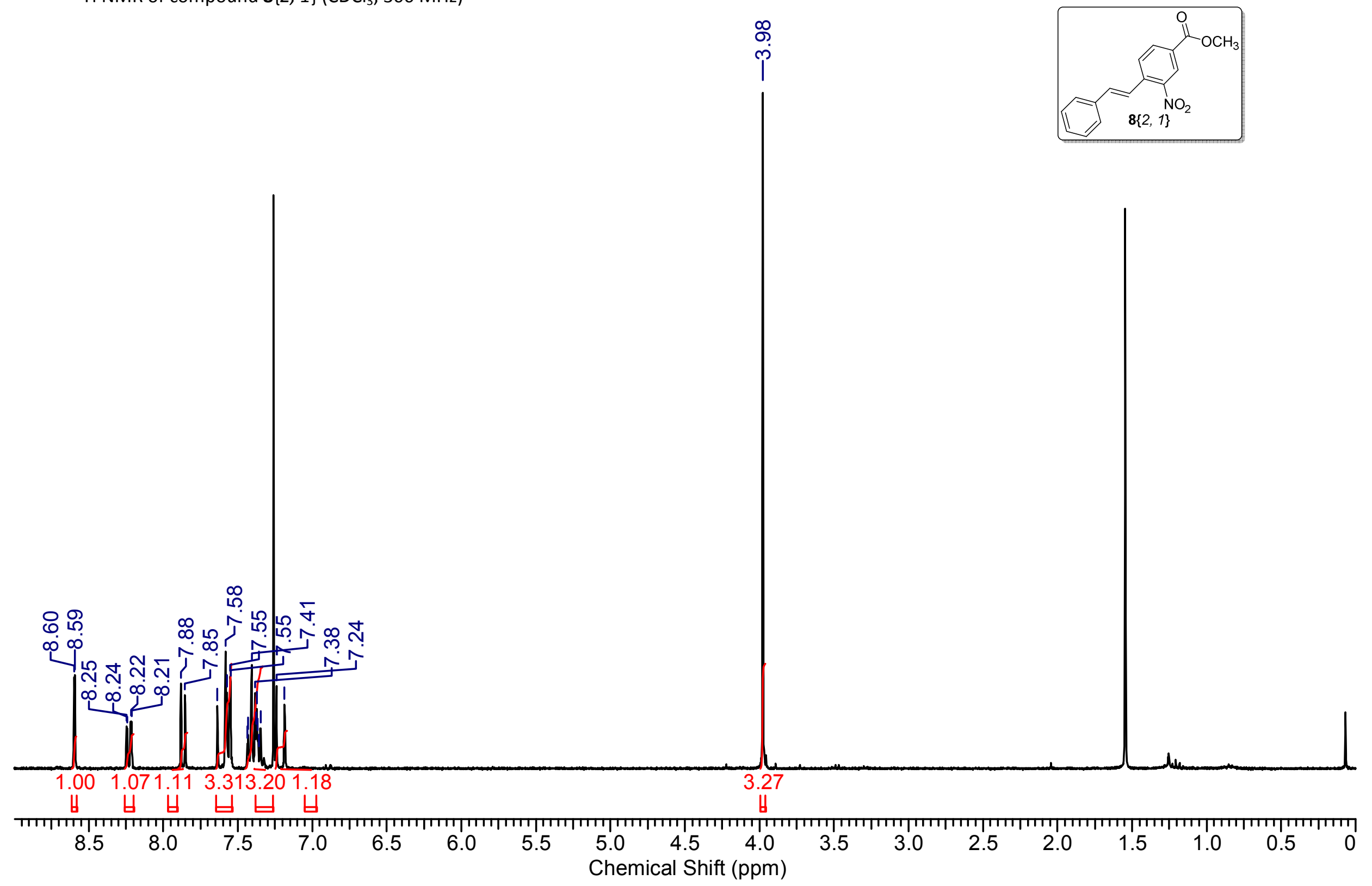


${ }^{13} \mathrm{C}$ NMR of compound $\mathbf{8}\{2,1\}\left(\mathrm{CDCl}_{3}, 75 \mathrm{MHz}\right)$
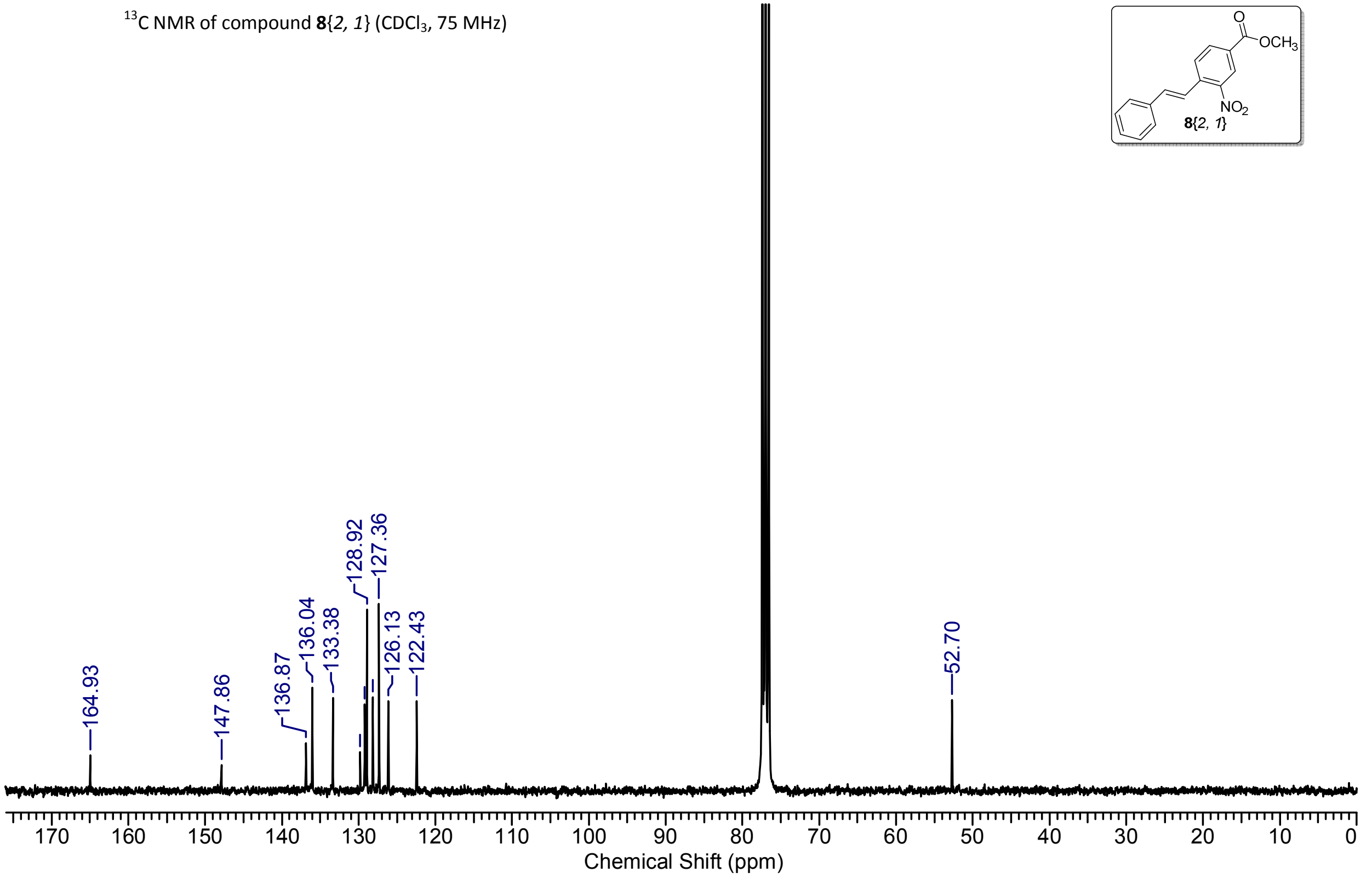
${ }^{1} \mathrm{H}$ NMR of compound $8\{1,4\}\left(\mathrm{CDCl}_{3}, 300 \mathrm{MHz}\right)$

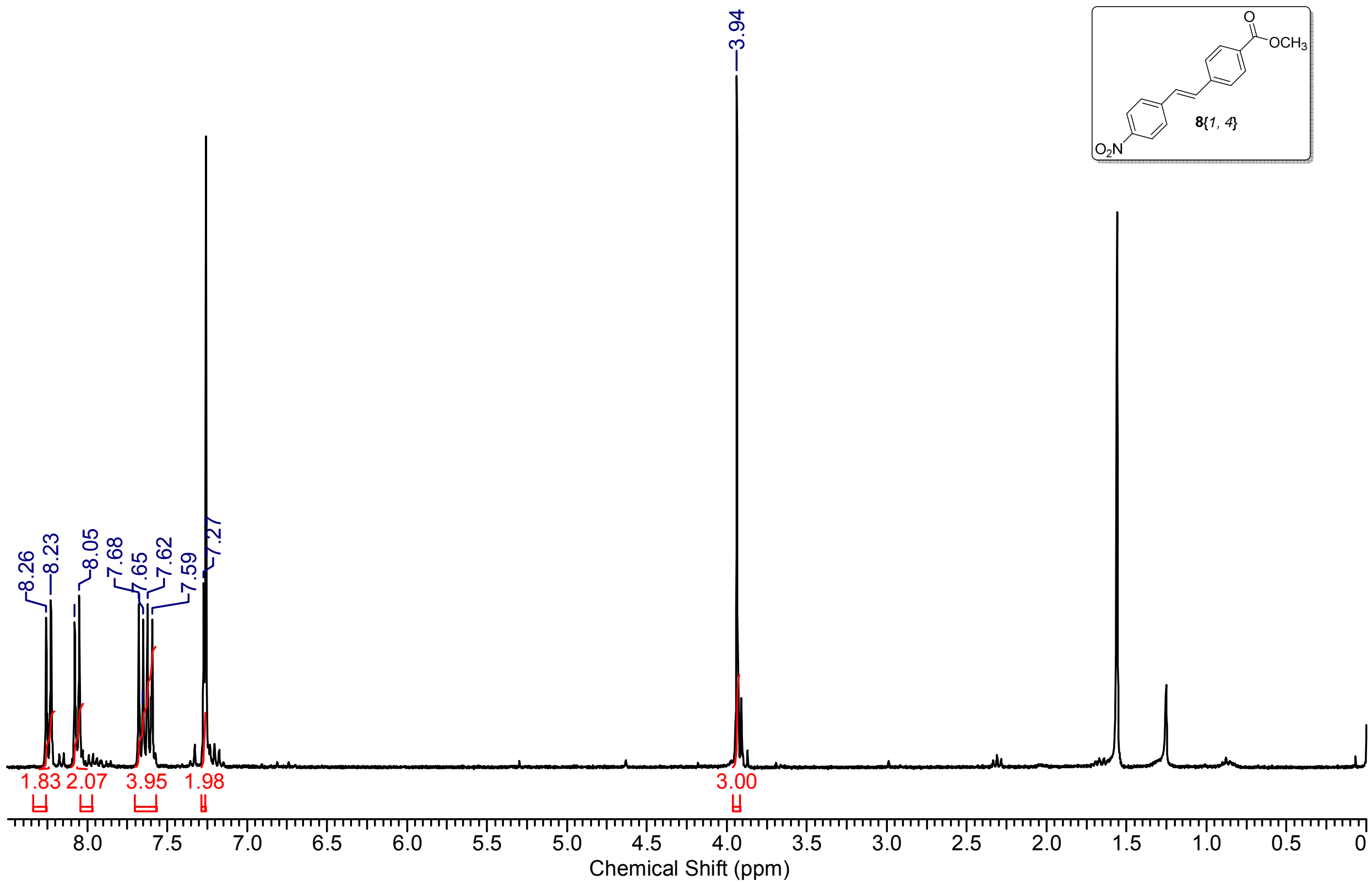


${ }^{13} \mathrm{C} \mathrm{NMR}$ of compound $8\{1,4\}\left(\mathrm{CDCl}_{3}, 75 \mathrm{MHz}\right)$
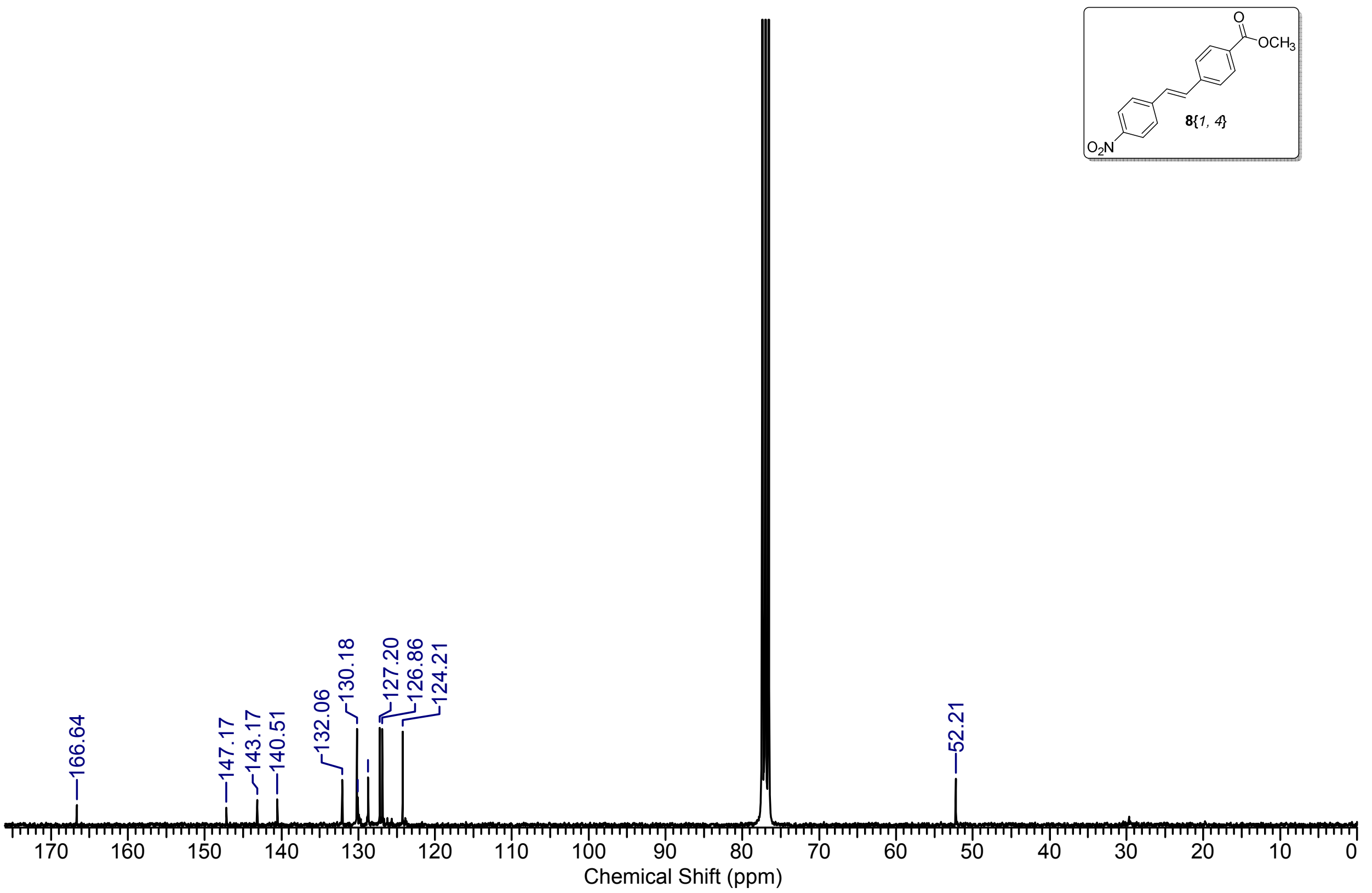
${ }^{1} \mathrm{H}$ NMR of compound $8\{1,5\}\left(\mathrm{CDCl}_{3}, 300 \mathrm{MHz}\right)$

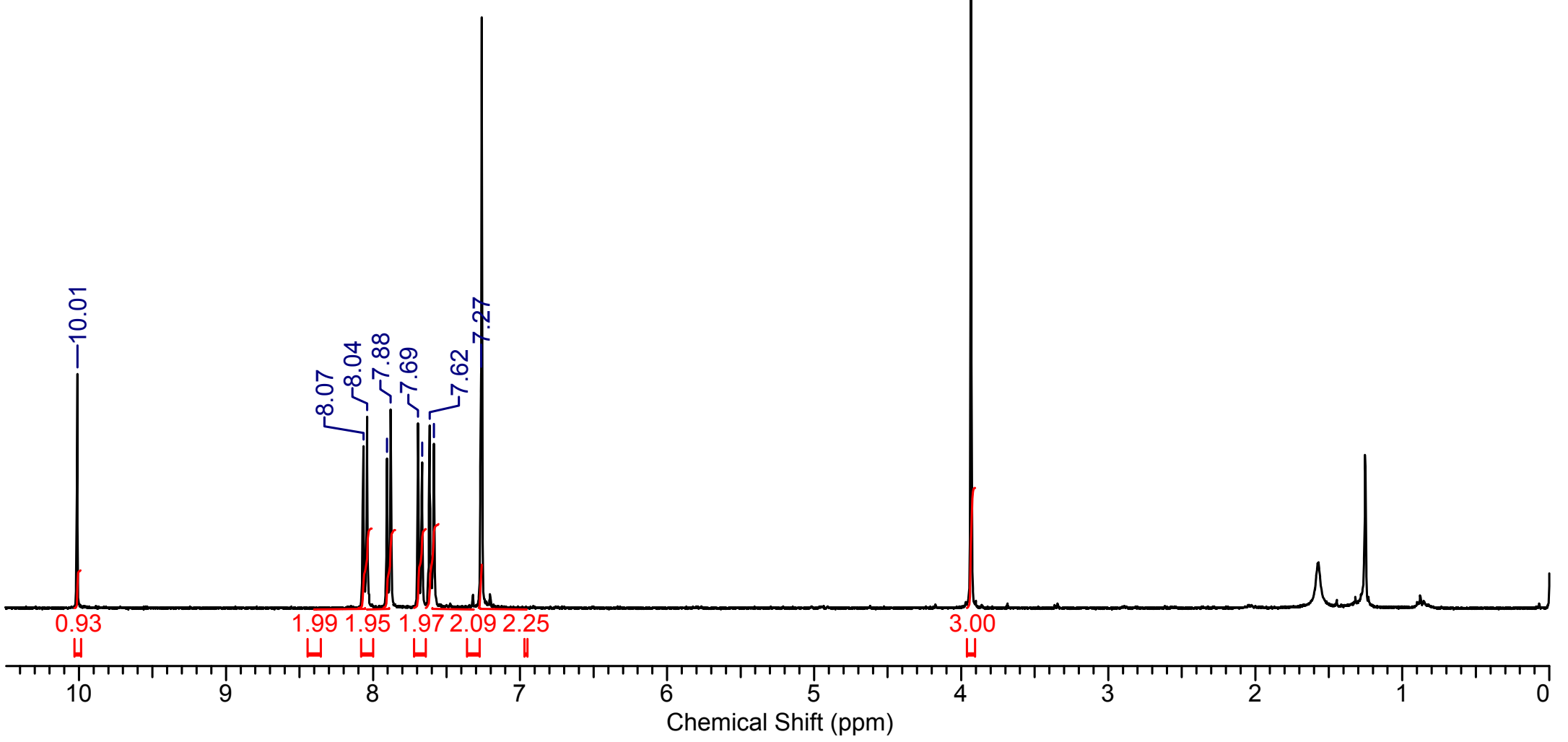


S18

${ }^{13} \mathrm{C} \mathrm{NMR}$ of compound $8\{1,5\}\left(\mathrm{CDCl}_{3}, 75 \mathrm{MHz}\right)$

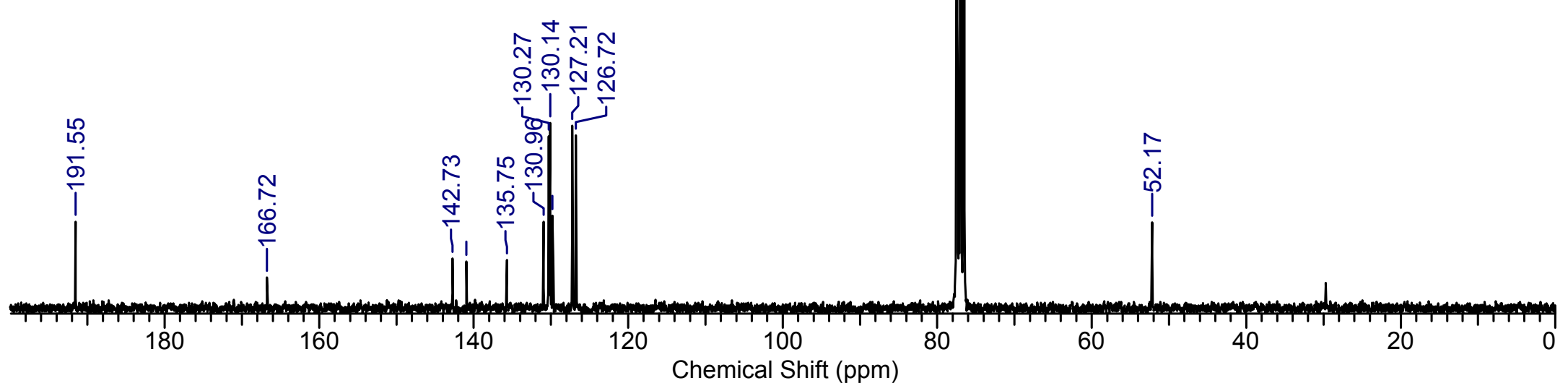


${ }^{1} \mathrm{H}$ NMR of compound $8\{1,6\}\left(\mathrm{CDCl}_{3}, 300 \mathrm{MHz}\right)$

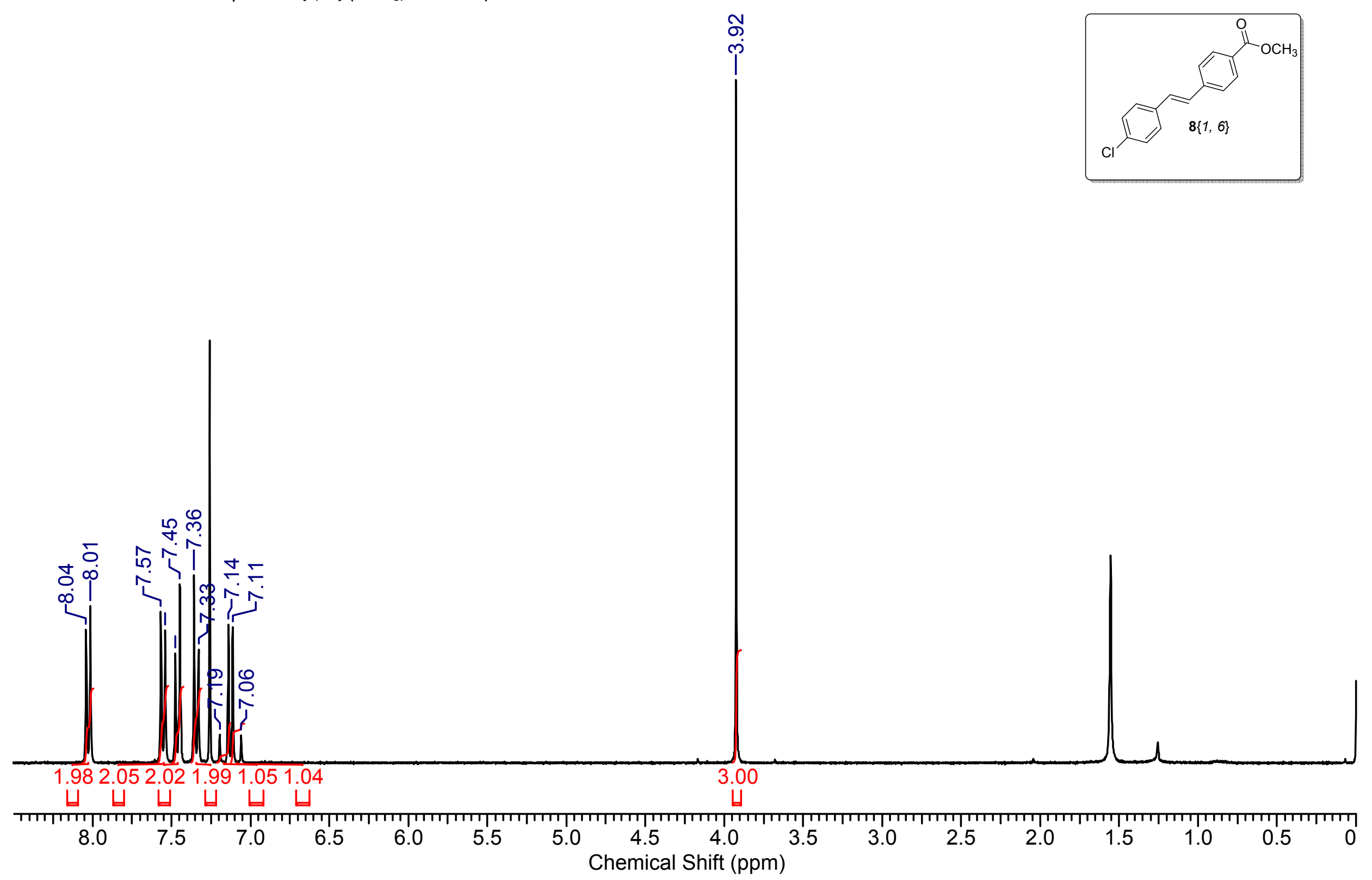


${ }^{13} \mathrm{C}$ NMR of compound $8\{1,6\}\left(\mathrm{CDCl}_{3}, 75 \mathrm{MHz}\right)$

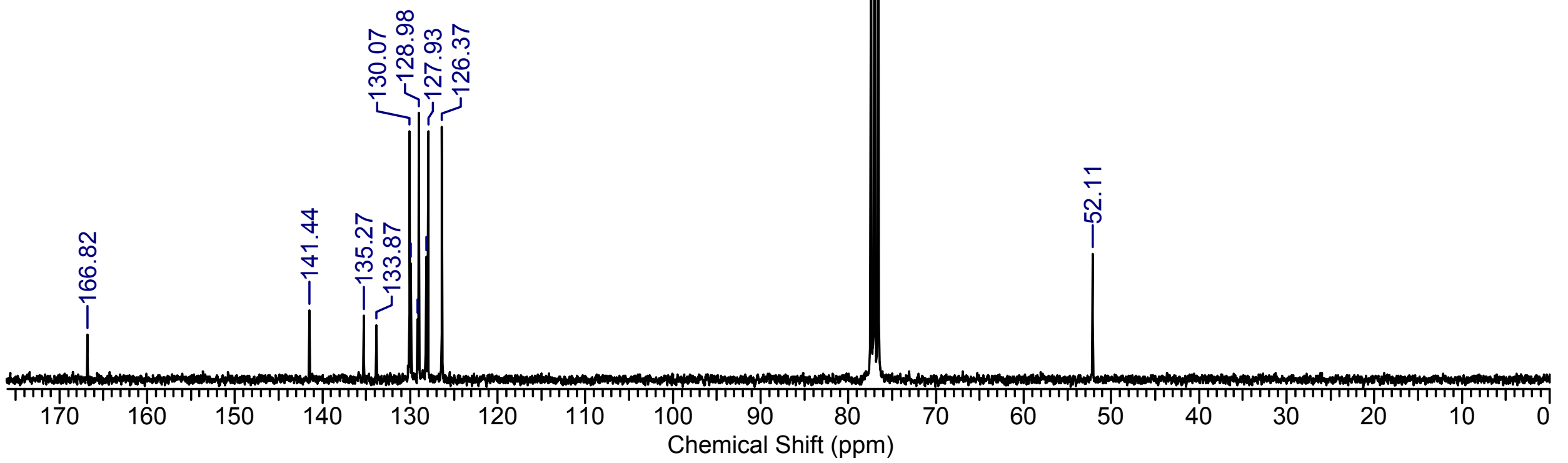


${ }^{1} \mathrm{H}$ NMR of compound $8\{2,2\}\left(\mathrm{CDCl}_{3}, 300 \mathrm{MHz}\right)$

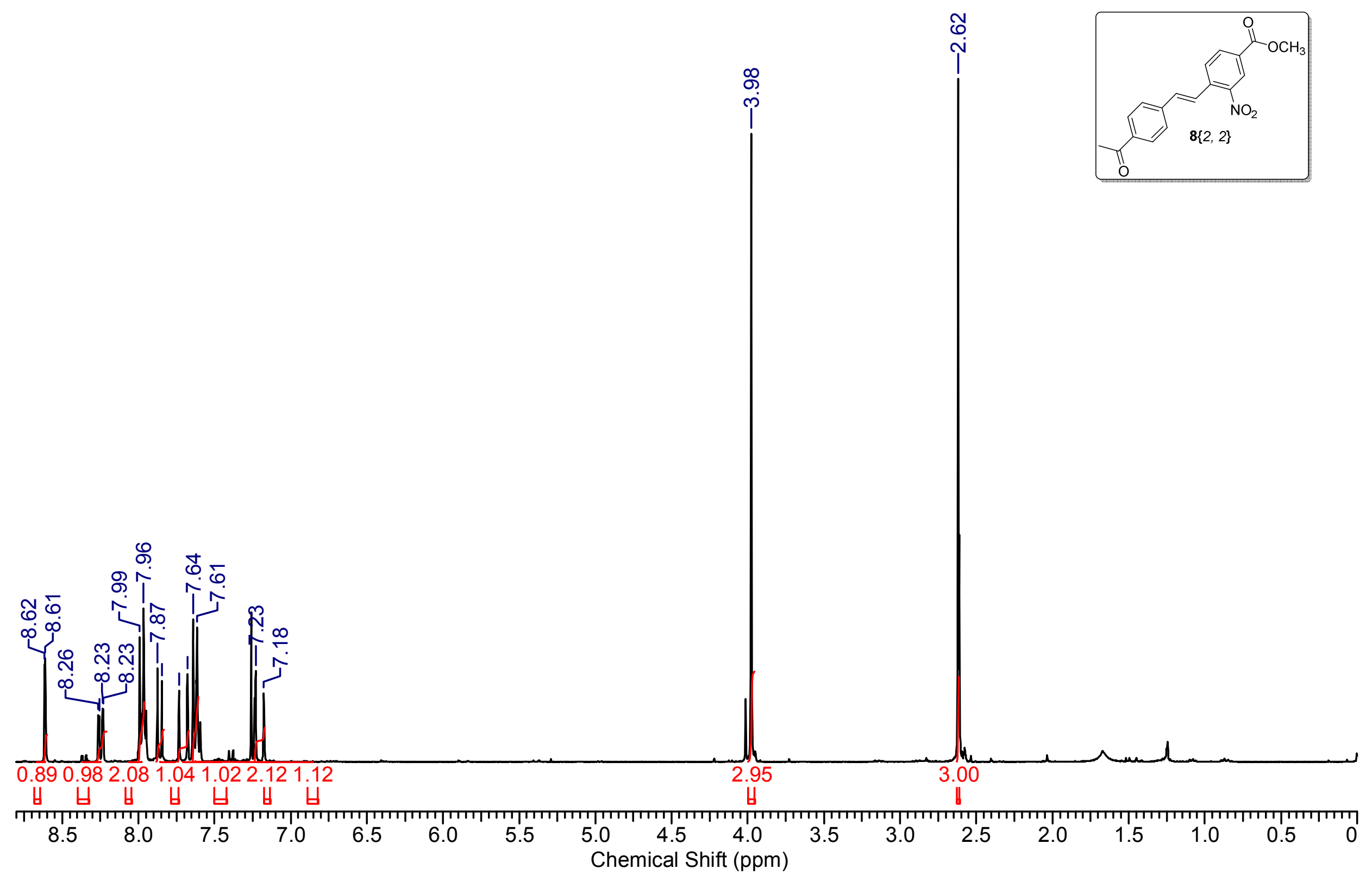


${ }^{13} \mathrm{C}$ NMR of compound $8\{2,2\}\left(\mathrm{CDCl}_{3}, 75 \mathrm{MHz}\right)$

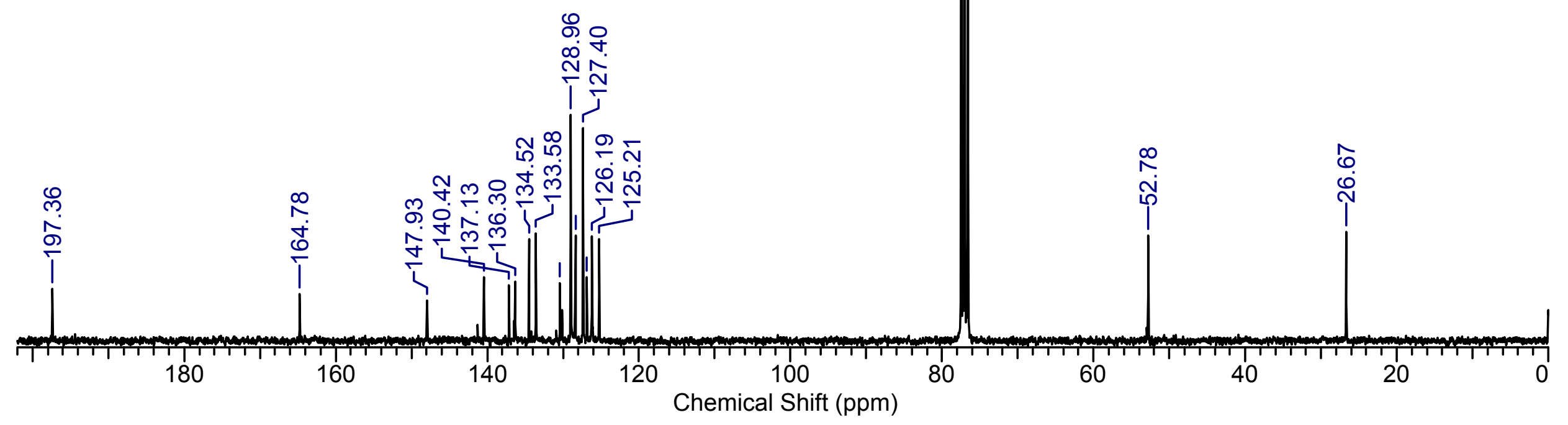


${ }^{1} \mathrm{H}$ NMR of compound $\mathbf{8}\{1,7\}\left(\mathrm{CDCl}_{3}, 300 \mathrm{MHz}\right)$

๓

i

(1)

$0.970 .982 .02 \quad 1.052 .081 .042 .08$

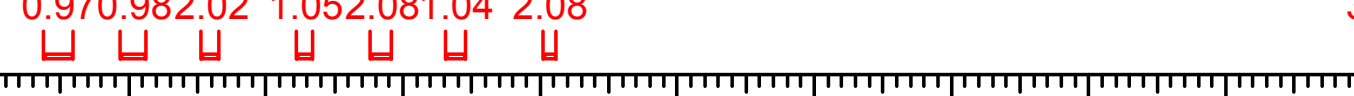

8.5

8.0

7.5

7.0

6.5

6.0

5.5

5.0

$4.5 \quad 4.0$

4.0
ppm $)$

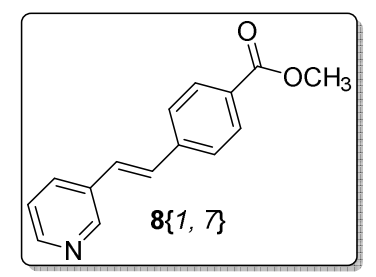

1

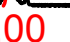


${ }^{13} \mathrm{C}$ NMR of compound $8\{1,7\}\left(\mathrm{CDCl}_{3}, 75 \mathrm{MHz}\right)$
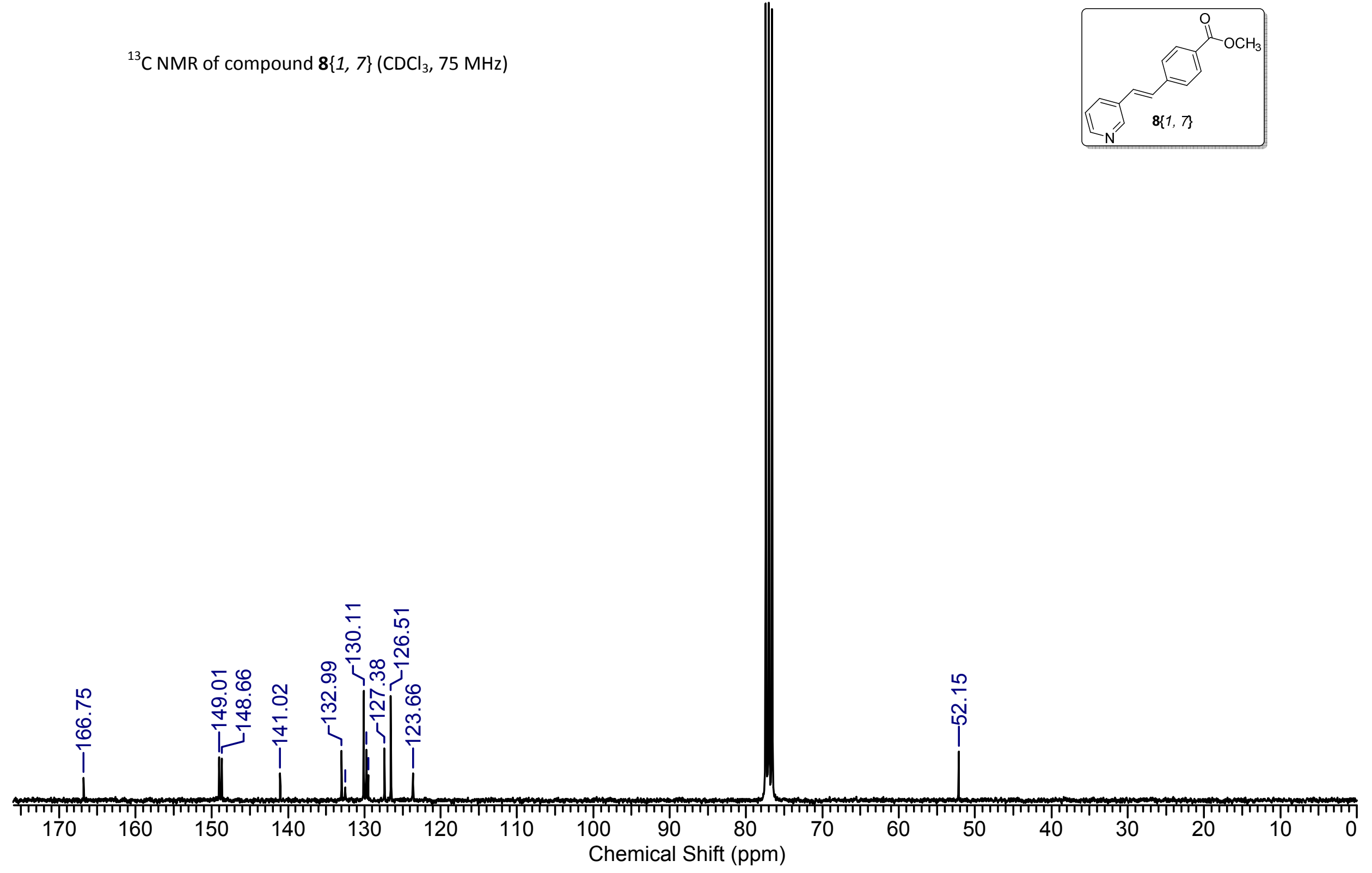
${ }^{1} \mathrm{H}$ NMR of compound $8\{2,3\}\left(\mathrm{CDCl}_{3}, 300 \mathrm{MHz}\right)$

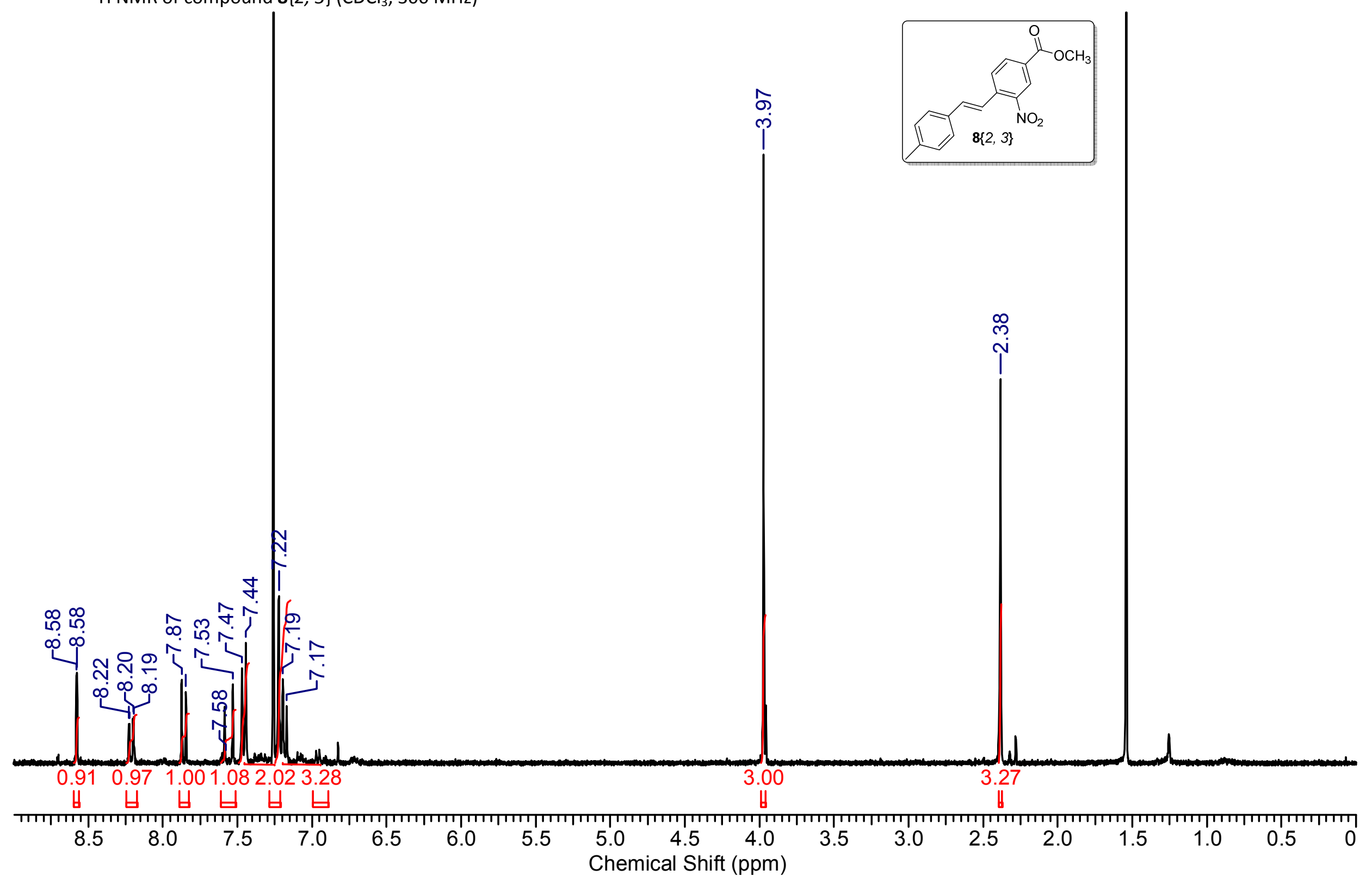


${ }^{13} \mathrm{CNMR}$ of compound $\mathbf{8}\{2,3\}\left(\mathrm{CDCl}_{3}, 75 \mathrm{MHz}\right)$
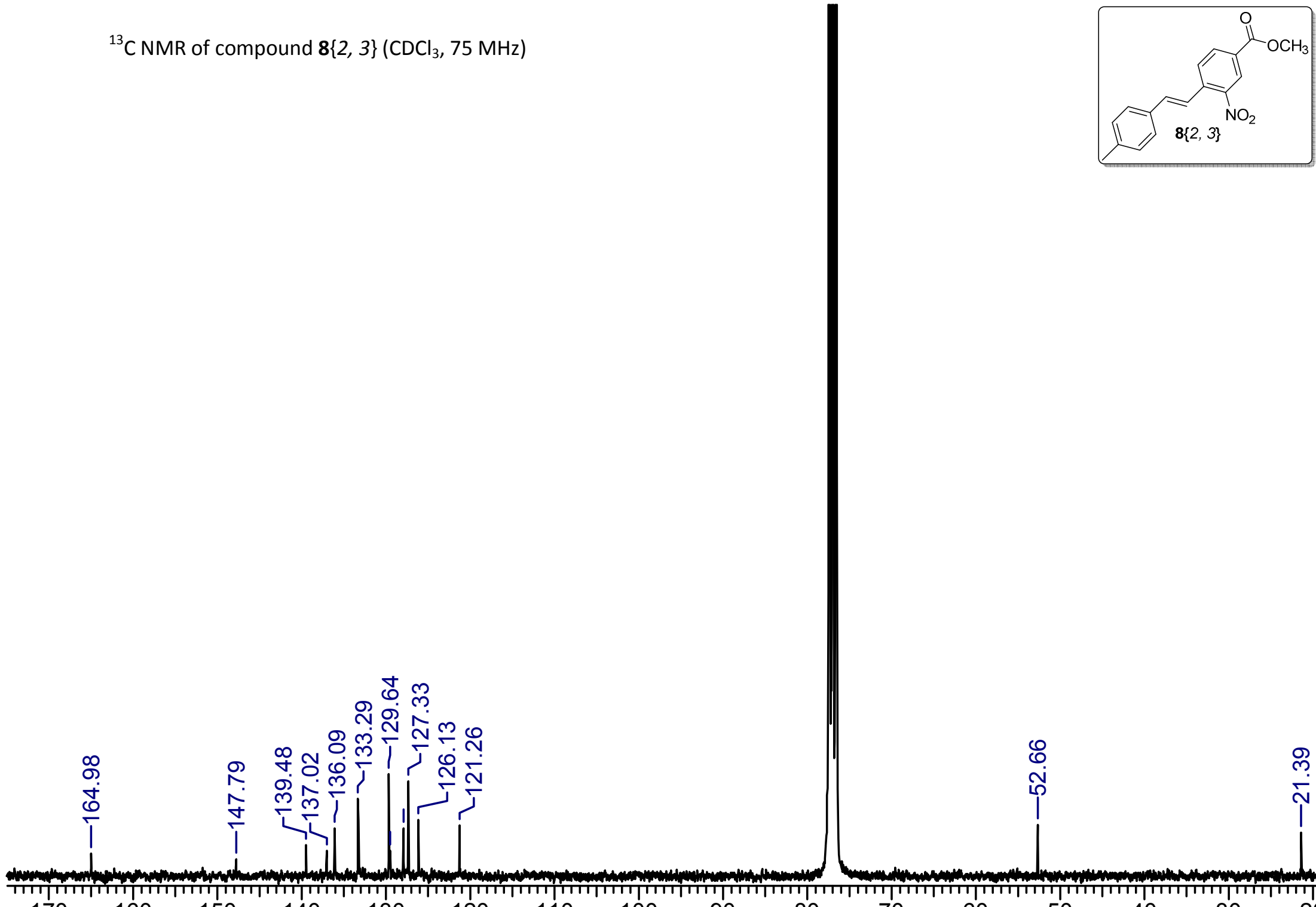

20 
${ }^{1} \mathrm{H}$ NMR of compound $8\{1,8\}\left(\mathrm{CDCl}_{3}, 300 \mathrm{MHz}\right)$

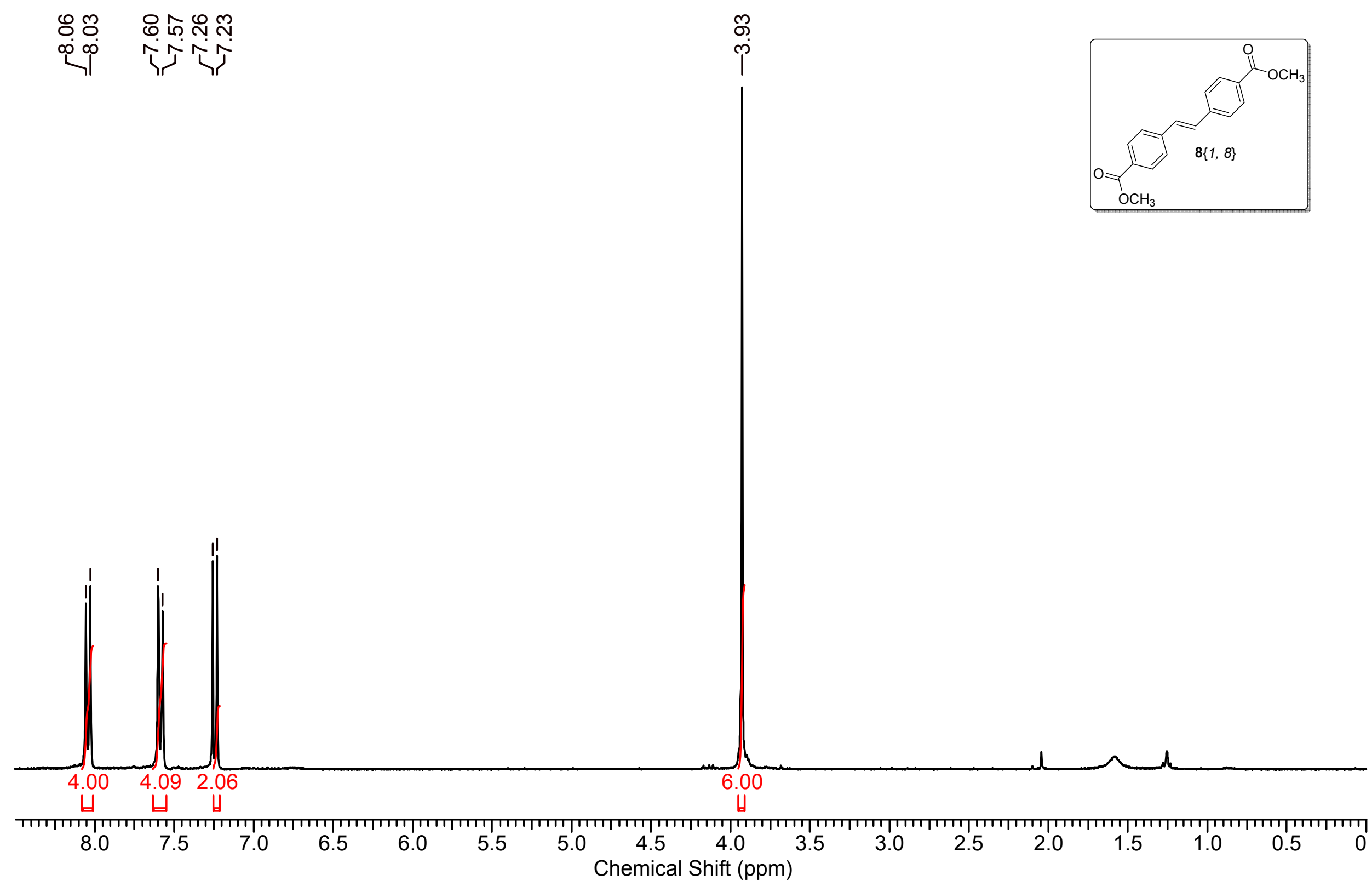


${ }^{13} \mathrm{C}$ NMR of compound $8\{1,8\}\left(\mathrm{CDCl}_{3}, 75 \mathrm{MHz}\right)$

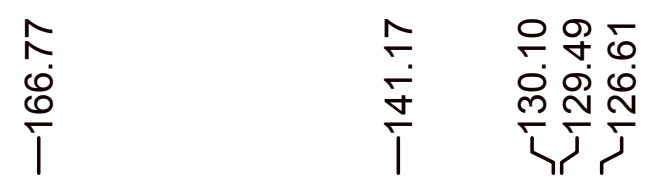

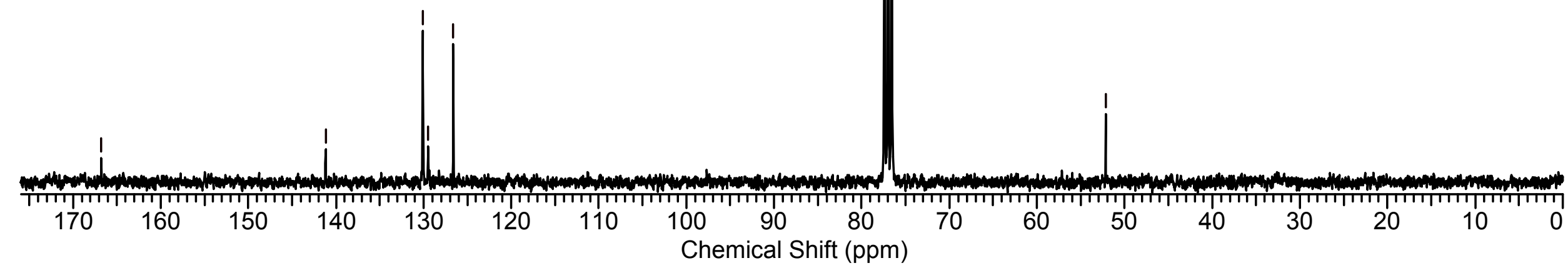


${ }^{1} \mathrm{H}$ NMR of compound $\mathbf{9}\{1,9\}\left(\mathrm{CDCl}_{3}, 300 \mathrm{MHz}\right)$
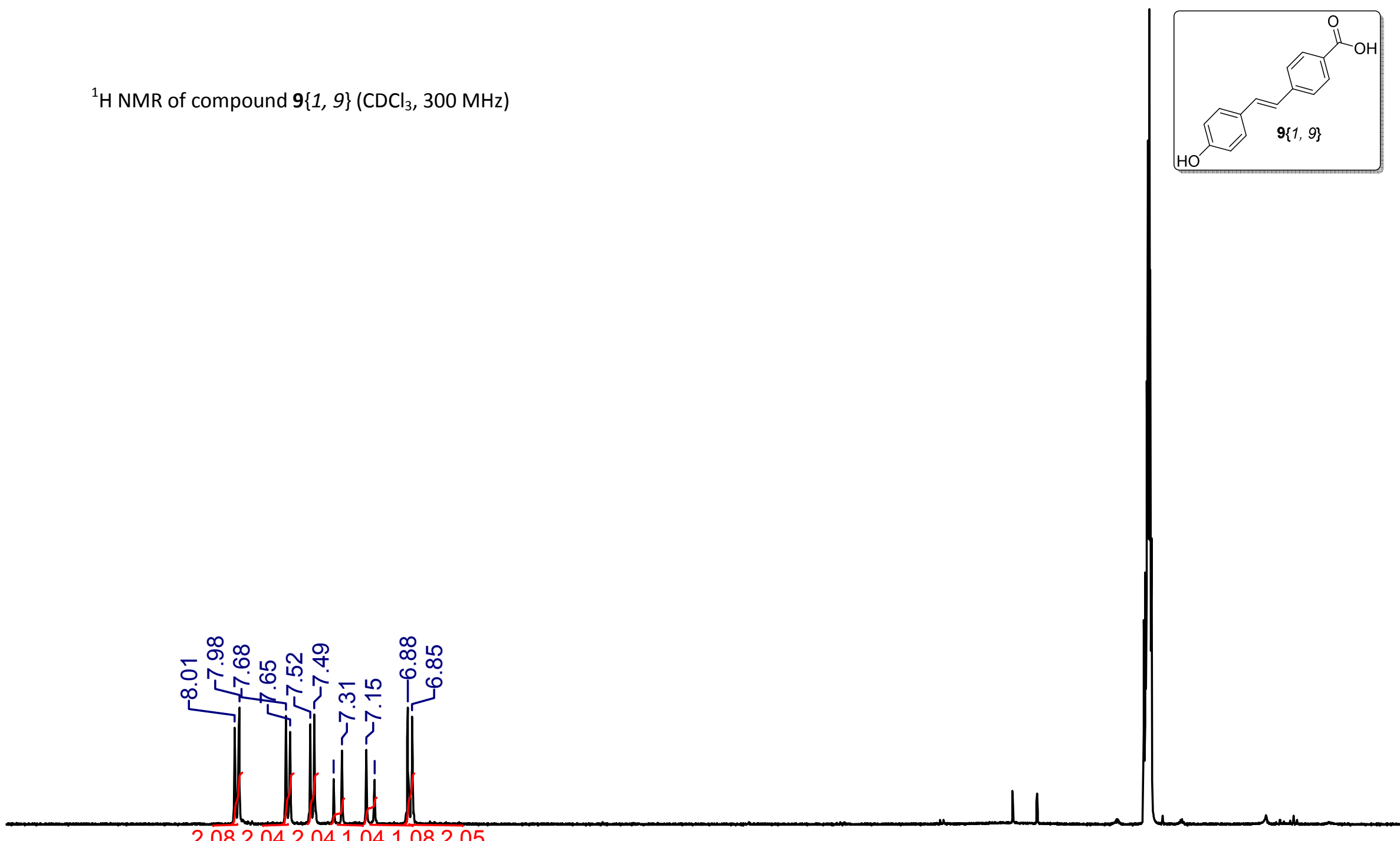

2.082 .042 .041 .041 .082 .05

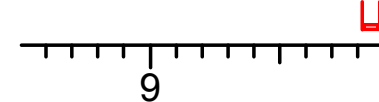

8

Chemical Shift (ppm) 
${ }^{13} \mathrm{C}$ NMR of compound $\mathbf{9}\{1,9\}\left(\mathrm{CDCl}_{3}, 75 \mathrm{MHz}\right)$

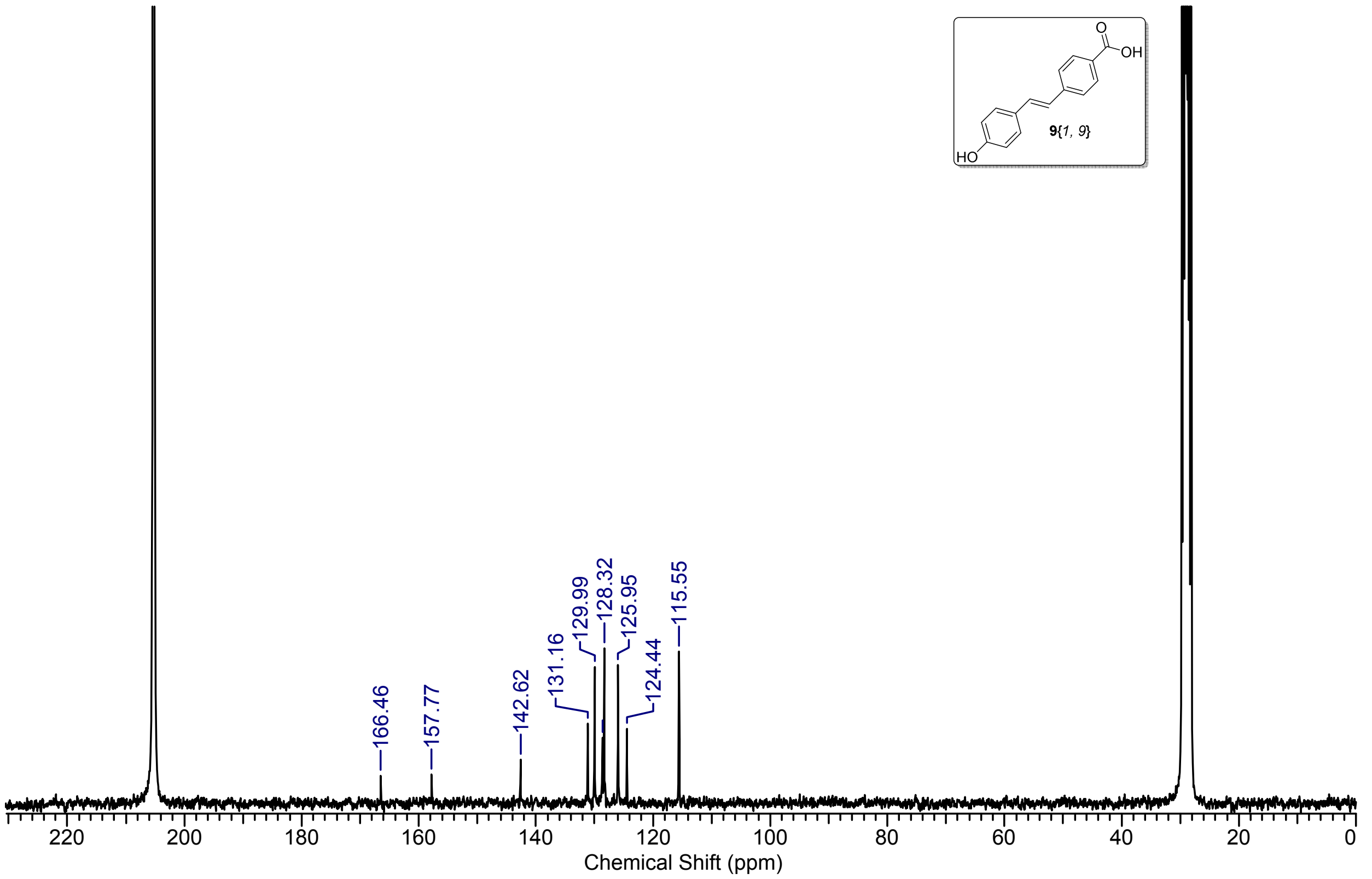


${ }^{1} \mathrm{H}$ NMR of compound $8\{1,10\}\left(\mathrm{CDCl}_{3}, 300 \mathrm{MHz}\right)$

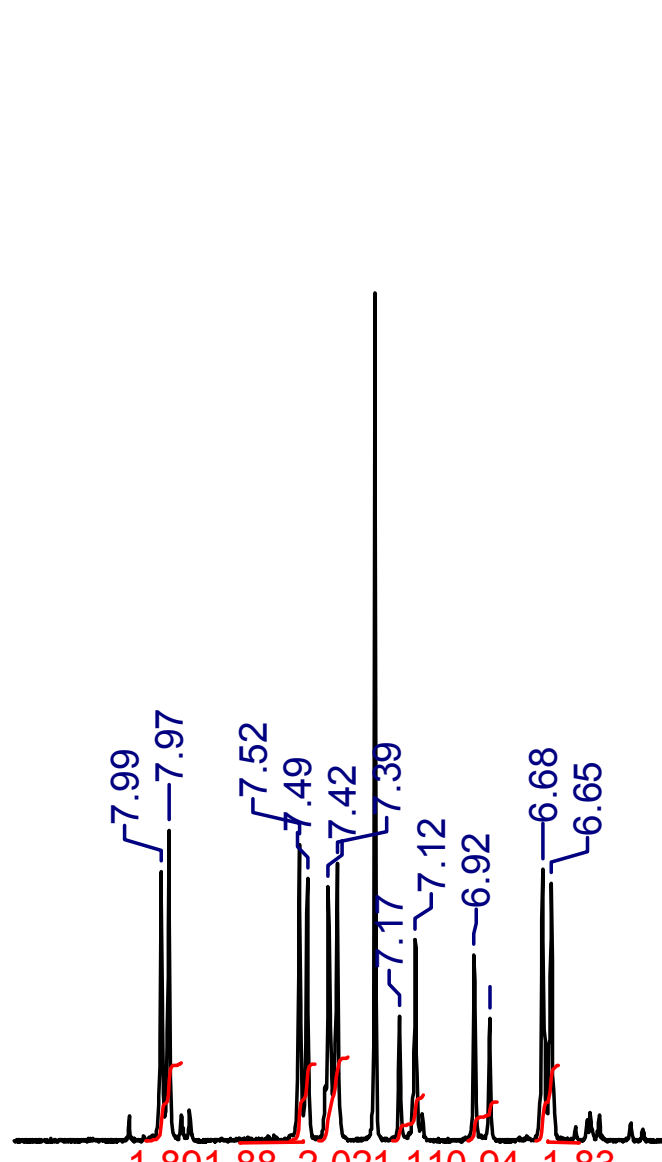

চூ

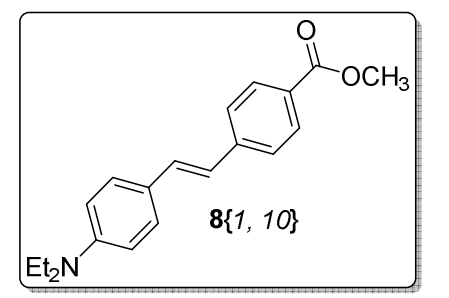

음

$\frac{1}{5}$

1.891 .88 2.021.110.94 1.83

ㅂㅂㅂ $ᄇ ᄇ ᄇ$

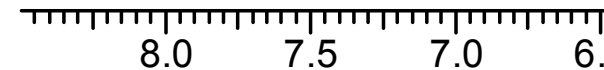
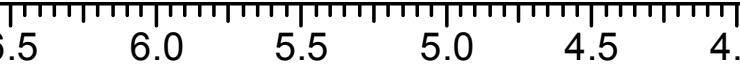

H

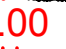

4.01

6.22

요

${ }^{2}$

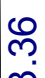

3.5

3.0

2.5

2.0 Chemical Shift (ppm) 
${ }^{13} \mathrm{C}$ NMR of compound $8\{1,10\}\left(\mathrm{CDCl}_{3}, 75 \mathrm{MHz}\right)$
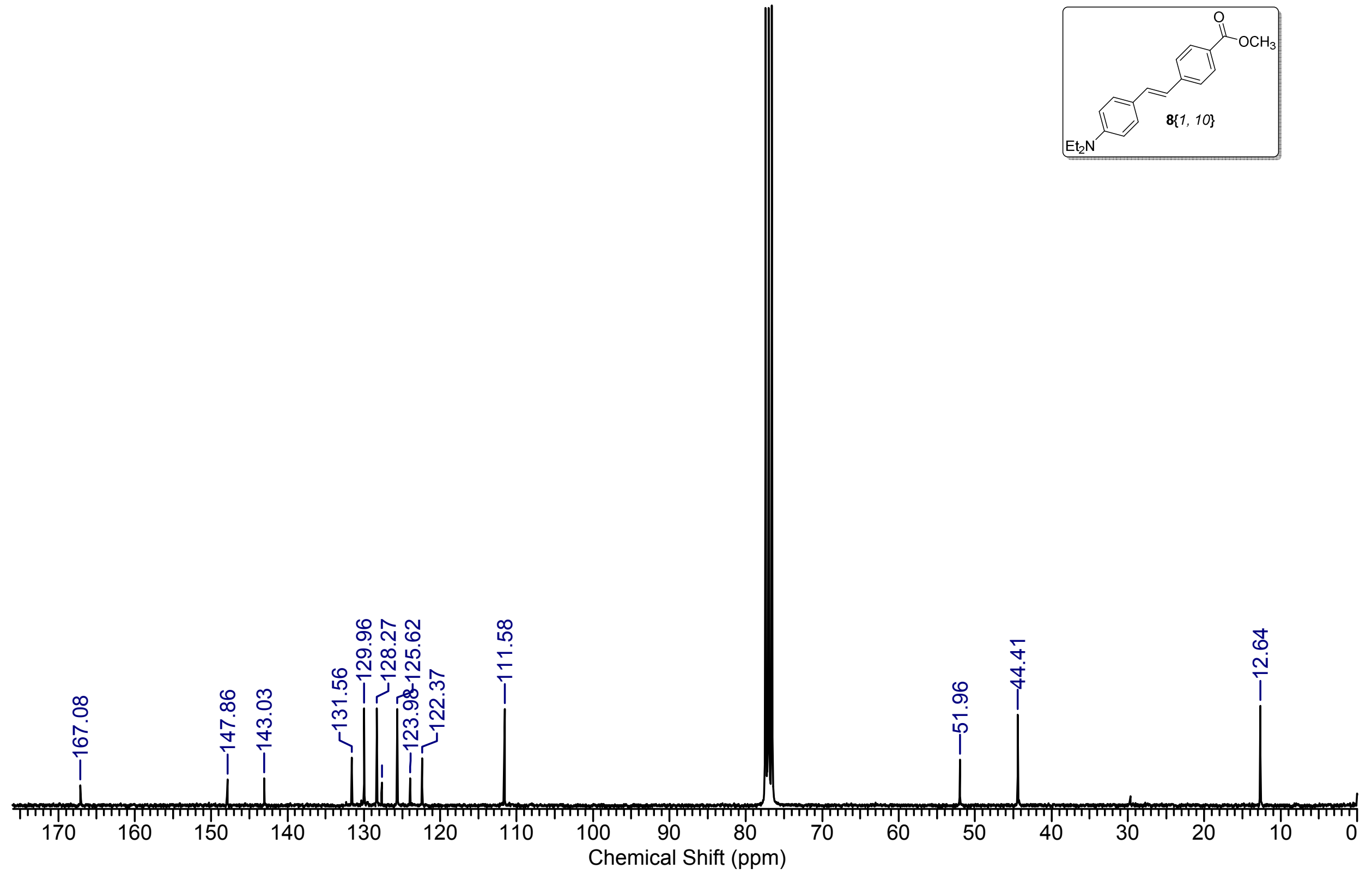


\section{${ }^{1} \mathrm{H}$ NMR of compound $\mathbf{8}\{1,11\}\left(\mathrm{CDCl}_{3}, 300 \mathrm{MHz}\right)$}

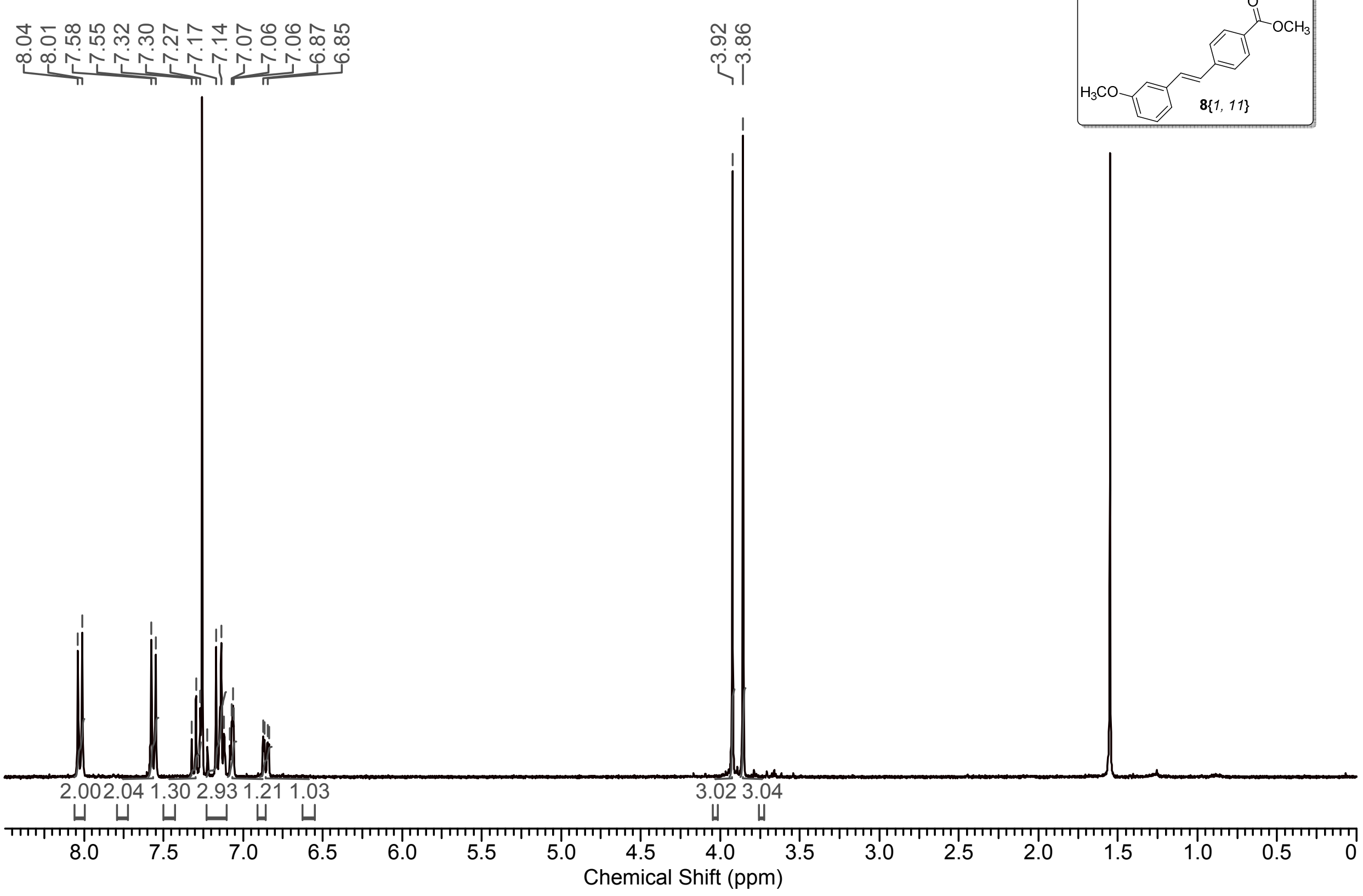


S34

${ }^{13} \mathrm{C}$ NMR of compound $8\{1,11\}\left(\mathrm{CDCl}_{3}, 75 \mathrm{MHz}\right)$

$\begin{array}{ll}\text { i } & \text { 1 } \\ 0 & 0 \\ 0 & 0 \\ 0 & 1\end{array}$

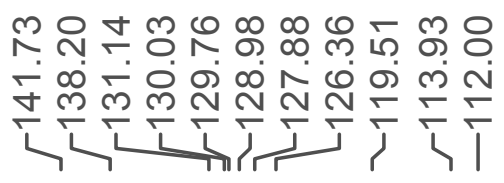

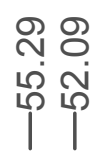
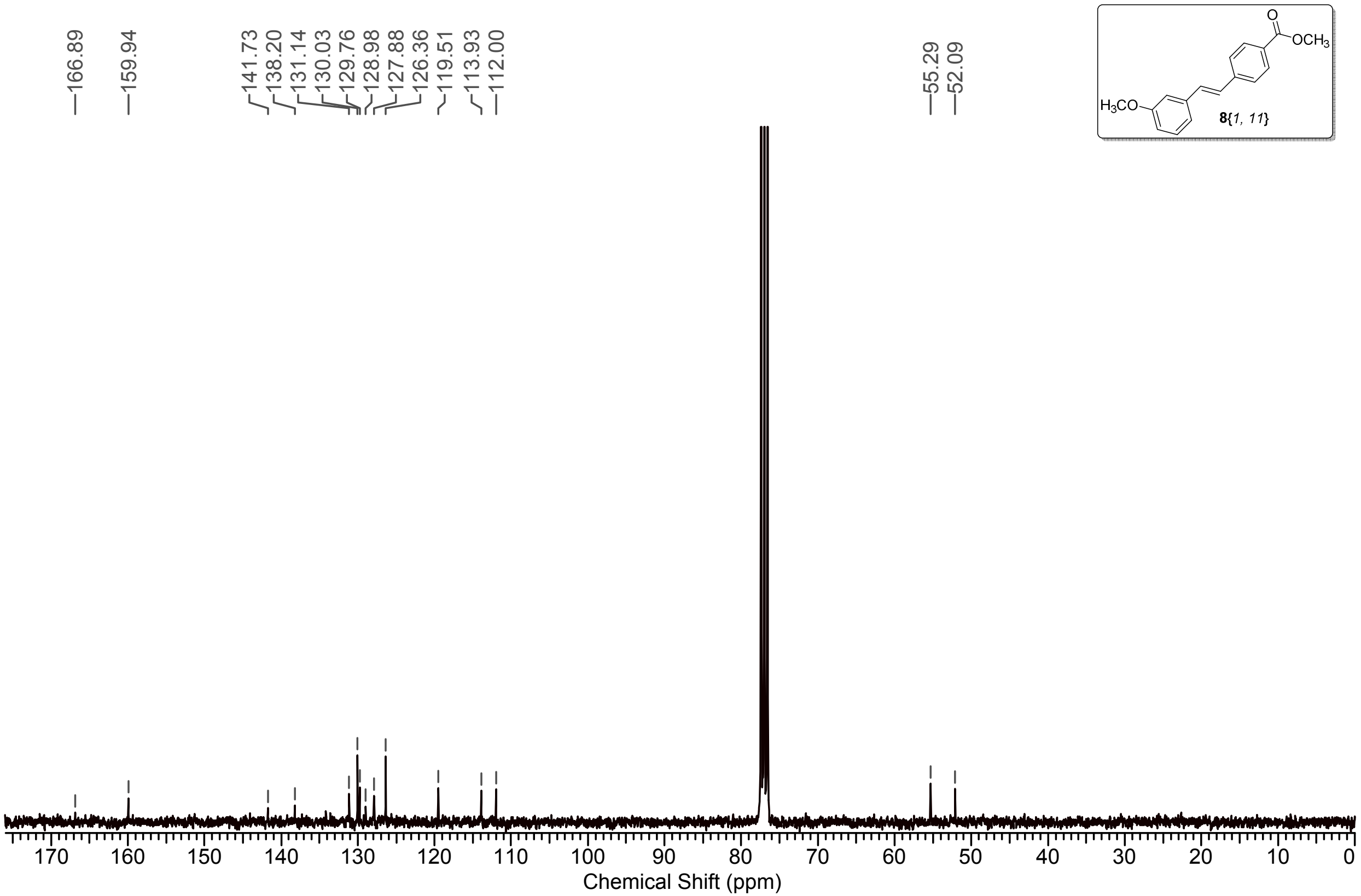